\title{
Building Abelian Functions with Generalised Baker-Hirota Operators
}

Matthew ENGLAND ${ }^{\dagger}$ and Chris ATHORNE $\ddagger$

${ }^{\dagger}$ Department of Computer Science, University of Bath, Bath, BA2 7AY, UK

E-mail: M.England@bath.ac.uk

URL: http://www.cs.bath.ac.uk/ me350/

$¥$ School of Mathematics and Statistics, University of Glasgow, G12 8QQ, UK

E-mail: Christopher.Athorne@glasgow.ac.uk

URL: www.gla.ac.uk/schools/mathematicsstatistics/staff/christopherathorne/

Received March 16, 2012, in final form June 18, 2012; Published online June 26, 2012

http://dx.doi.org/10.3842/SIGMA.2012.037

\begin{abstract}
We present a new systematic method to construct Abelian functions on Jacobian varieties of plane, algebraic curves. The main tool used is a symmetric generalisation of the bilinear operator defined in the work of Baker and Hirota. We give explicit formulae for the multiple applications of the operators, use them to define infinite sequences of Abelian functions of a prescribed pole structure and deduce the key properties of these functions. We apply the theory on the two canonical curves of genus three, presenting new explicit examples of vector space bases of Abelian functions. These reveal previously unseen similarities between the theories of functions associated to curves of the same genus.
\end{abstract}

Key words: Baker-Hirota operator; $\mathcal{R}$-function; Abelian function; Kleinian function

2010 Mathematics Subject Classification: 14H40; 14H50; 14H70

\section{Introduction}

The overall aim of this paper is the presentation of a systematic method of construction of Abelian functions on Jacobian varieties of plane, algebraic curves. Apart from its purely theoretical interest this is important from the point of view of at least two applications. Firstly, the relations between Abelian functions of given pole order, that is, the expansion of given functions in terms of the basis, are partial differential equations of the kind arising in integrable systems theory. In particular, members of the KP hierarchy have been shown to arise in this way, for functions associated with hyperelliptic curves of general genus and also for various nonhyperelliptic curves. Consequently, the Abelian functions constitute one class of solution to such partial differential equations. This connection is explicit even in the early work of Baker (see later references). Secondly, Abelian functions with shifted argument expanded in terms of the basis of functions in un-shifted arguments give addition laws generalizing the classic addition law of points on the cubic curve. This expresses the group structure of the Jacobian. It is also important in the theory of discrete integrable systems.

The need for a new systematic method of construction is motivated in Section 1.2 by the so called basis problem for Abelian functions. The solution we present is based on our definition of generalised Baker-Hirota operators, following the work of Baker on Abelian functions and the work of Hirota in soliton theory. Hence we continue the introduction in Section 1.1 with a discussion of the Hirota derivative and how it motivates the general operators which follow. 


\subsection{Hirota derivatives and operators}

The Hirota derivative is originally a device belonging to the direct method of solution to soliton equations, [27]. A simple paradigm is provided by the Korteweg-de Vries equation,

$$
u_{t}+u_{x x x}+6 u u_{x}=0 .
$$

Substitution for $u(x, t)$ by $2(\ln F(x, t))_{x x}$ and integration with respect to $x$ (neglecting arbitrary functions of $t$ ) yields the bilinear form

$$
D_{x}\left(D_{t}+D_{x}^{3}\right) F \cdot F=0,
$$

where the Hirota derivative is defined as

$$
D_{x}^{n} D_{t}^{m} F \cdot G=\left.\partial_{x^{\prime}}^{n} \partial_{t^{\prime}}^{m} F\left(x+x^{\prime}, t+t^{\prime}\right) G\left(x-x^{\prime}, t-t^{\prime}\right)\right|_{x^{\prime}=0, t^{\prime}=0} .
$$

Thus, for example,

$$
\begin{aligned}
& D_{x} F \cdot G=F_{x} G-F G_{x}, \\
& D_{x} D_{t} F \cdot G=F_{x t} G-F_{x} G_{t}-F_{t} G_{x}+F G_{x t}
\end{aligned}
$$

etc. The $N$-soliton solutions arise via an ansatz of the form $F(x, t)=f\left(e^{\theta_{1}}, e^{\theta_{2}}, \ldots, e^{\theta_{N}}\right)$ where the $\theta_{i}$ are linear in $x$ and $t$.

Of course, the Hirota "derivative" is not actually a derivative since it does not act on products but on pairs as signalled by the above. However, if we consider a product on function pairs of the kind $(f, g)(h, k)=(f h, g k)$ and define $\mathcal{D}_{x}$ etc. as Hirota operators that map from function pairs to function pairs,

$$
\mathcal{D}_{x}(f, g)=\left(\partial_{x} f, g\right)-\left(f, \partial_{x} g\right)
$$

then we do have a Leibniz derivation formula:

$$
\mathcal{D}_{x}((f, g)(h, k))=\mathcal{D}_{x}(f, g)(h, k)+(f, g) \mathcal{D}_{x}(h, k) .
$$

Because further, this Hirota operator is bilinear over addition and scalar multiplication, it is sensible to treat the function pairs as tensor products and the product as the standard multiplication on tensor products:

$$
(f \otimes g)(h \otimes k)=f h \otimes g k .
$$

The original Hirota derivative is then a composition of $\mathcal{D}_{x}$ with a total symmetrization operator, $S$, mapping from the tensor to the differential polynomial algebra:

$$
\begin{array}{ccccc}
F \otimes F & \stackrel{\mathcal{D}_{x}}{\longrightarrow} & F \otimes F & \stackrel{S}{\longmapsto} & F, \\
f \otimes g & \stackrel{\mathcal{D}_{x}}{\longmapsto} & f_{x} \otimes g-f \otimes g_{x} & \stackrel{S}{\longmapsto} & f_{x} g-f g_{x} .
\end{array}
$$

Here $F$ denotes a $\mathfrak{k}$-algebra of appropriate $\mathfrak{k}$-valued functions, for some field $\mathfrak{k}$.

Such a generalisation has been the subject of previous work by one of the authors, applied to invariant theory [2], the theory of Padé approximants [1] and to generalised $\wp$-functions [5].

It is also interesting to compare with algebraic notions of derivations over tensor products of algebras introduced in [31]. It is not clear to the present authors whether $\mathcal{D}$ is either an example or a generalization of this approach but it suggests an avenue for further investigation

In each of these applications, there is an equivariance property of the Hirota operators appropriate to the case. Suppose we have a group, $\mathcal{G}$ acting on the space of independent variables 
represented by the list $x$. We assume there is an induced representation of $\mathcal{G}$ on some set of functions of $x$ :

$$
f\left(G^{-1} x\right)=f^{G}(x) .
$$

In some applications $\mathcal{G}$ is a Lie group and we are interested in a Lie algebra representation. Then a Hirota operator $\mathcal{D}$, (we drop the subscript), should have the desirable property:

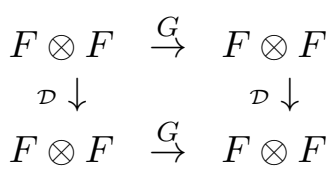

In the case of the Abelian functions associated to a curve, just such an action is obtained. Given a curve of genus $g$ we define the associated Abelian functions as those meromorphic functions of $g$ complex variables $\boldsymbol{u} \in \mathbb{C}^{g}$, which are periodic with respect to the period lattice of the curve. All such functions may be expressed algebraically using the $\sigma$-function of the curve. A translation across lattice points, $\boldsymbol{u} \mapsto \boldsymbol{u}+\boldsymbol{\ell}$ induces a multiplicative factor: $\sigma(\boldsymbol{u}+\boldsymbol{\ell})=h(\boldsymbol{u}, \boldsymbol{\ell}) \sigma(\boldsymbol{u})$. The generalised Hirota operators we introduce here will generate Abelian functions out of tensor products of these $\sigma$-functions.

Our generalisation of the Hirota operator picks up from earlier work. In [24], trilinear Hirota derivatives are presented in the context of certain integrable equations in trilinear form. They are characterised by a simple equivariance property. Further work can be found in $[25,26]$.

In [2] a more algebraic, independent treatment is developed for Hirota type operators acting on arbitrary tensor products of finite-dimensional $\mathfrak{s l}_{2}(\mathbb{C})$-modules. In the infinite dimensional limit the $\mathfrak{s l}_{2}$ action becomes a Heisenberg action and the Hirota operators become the familiar Hirota derivatives (when acting on degree two tensor products). This interplay has also been exploited in a study of differential normal forms, [34].

In the present paper we deal with arbitrary tensor products of a differential algebra over some field, $\mathfrak{k}$, which may as well be $\mathbb{C}$ given the analytic context. The differential algebra is generated by a Kleinian $\sigma$-function in variables $u_{1}, \ldots, u_{g}$ and its partial derivatives. There is a Heisenberg action on the derivatives with respect to each individual $u_{i}$ and an $\mathfrak{s l}_{2}(\mathbb{C})$ action on the whole set of $u_{i}$. For example the set of first order derivatives $\left(\partial_{u_{1}} \sigma, \partial_{u_{2}} \sigma, \ldots, \partial_{u_{g}} \sigma\right)$ is a basis for a $g$ dimensional $\mathfrak{s l}_{2}(\mathbb{C})$-module.

\subsection{The basis problem for Abelian functions}

The main motivation for the generalised Hirota operators presented in this paper is to solve the so called basis problem for Abelian functions. The simplest Abelian functions are the elliptic functions, associated with elliptic curves, which of course have genus one. Elliptic functions have been the subject of much study since their discovery and have been extensively used to enumerate solutions of non-linear wave equations. They occur in many physical applications; traditionally the arc-length of the lemniscate and the dynamics of spherical pendulums, [32], but also in cryptography, [36], and soliton solutions to the KdV equation, [14].

Recent times have seen a revival of interest in the theory of their generalisations. These Abelian functions are also beginning to find a wide range of applications. For example, they give further solutions to the $\mathrm{KdV}$ equation along with solutions to other integrable equations from the KP-hierarchy (see for example [9, 11, 21]). They have also been used to describe geodesic motions in certain space-time metrics, [23].

Much progress has been made through the realisation of Abelian functions as generalisations of the Weierstrass $\wp$-function, following the approach of Klein and Baker for hyperelliptic functions. In the genus $g$ case the original Weierstrass $\wp$-function is generalised to a family of 
functions denoted $\wp_{i j}(\boldsymbol{u})$ for $i, j \in(1, \ldots, g)$. It is the case that $\wp_{i j}=\wp_{j i}$ and so there are $\frac{1}{2} g(g+1)$ functions in total. We have the integrability property that

$$
\frac{\partial}{\partial u_{i}} \wp_{j k}=\frac{\partial}{\partial u_{j}} \wp_{i k}
$$

So we can denote the derivatives of the functions using additional indices and adopt the notation of writing the indices in ascending numerical order. The 3 -index $\wp$-functions generalise the derivative of the Weierstrass function, $\wp^{\prime}$ and so on.

We may classify the Abelian functions according to their pole structure. The $m$-index $\wp$ functions will have poles of order $m$ for example. We can define a vector space of Abelian functions with poles of at most a given order and so the question of building bases for the vector spaces naturally arises. The Riemann-Roch theorem for Abelian varieties gives the dimension of the vector space as $m^{g}$ where $m$ is the maximal pole order. In the genus one case each successive vector space will have dimension one higher, and the new basis function can be played by the next derivative of the $\wp$-function. However, for $g>1$ there are not enough $\wp$-functions to build the bases. Indeed, as both $g$ and $m$ increase so does the deficit of functions. This is the basis problem.

Solutions to the problem have been found in a number of specific cases. In genus 2 the introduction of one further function with poles of order 3 is sufficient to allow differentiation to generate all subsequent bases. This new function is the difference $\wp_{11} \wp_{22}-\wp_{12}^{2}$, in which the poles of order 4 in each term cancel. In $[16,19]$ the basis problem was solved in various cases, by a group including one of the present authors, through the introduction of analogous functions; polynomials in $\wp$ with coefficients chosen to cancel poles. However for curves with $g>2$ hyperelliptic and $g>3$ non-hyperelliptic the bases cannot be finitely generated by differentiation and this approach does not generalise easily to give an infinite number of functions.

Solving the basis problem makes it easier to work with the functions in a wide range of applications. For example, in [22] a reduction for the Benney equation was constructed using the Abelian functions associated with a genus six curve and having a basis for the functions limited the search for the explicit form of the reduction. The bases can also be used to derive more theoretical results, such as the differential equations satisfied by the functions. These can be derived from a set which generalise the classic differential equation for the Weierstrass $\wp$-function,

$$
\left(\wp^{\prime}(u)\right)^{2}=4 \wp(u)^{3}-g_{2} \wp(u)-g_{3} .
$$

The bases can also be used to construct various addition formulae for the functions. For example, there are generalisations of the following well known addition formula for the Weierstrass functions,

$$
-\frac{\sigma(u+v) \sigma(u-v)}{\sigma(u)^{2} \sigma(v)^{2}}=\wp(u)-\wp(v) .
$$

Each generalisation has the same ratio of $\sigma$-functions expressed as a polynomial in an appropriate basis of Abelian functions. For curves with an extra cyclic symmetry in their coordinates there are other classes of addition formulae. For example, suppose the curve is invariant under $\left[\zeta^{j}\right]:(x, y) \rightarrow\left(x, \zeta^{j} y\right)$ where $\zeta$ is some primitive $m$ th root of unity. Then we can define a corresponding action on the variables $\boldsymbol{u}$ and find an expression in an appropriate basis of Abelian functions for

$$
\prod_{j=1}^{m} \frac{\sigma\left(\sum_{i=1}^{m}\left[\zeta^{i+j}\right] \boldsymbol{u}^{[i]}\right)}{\sigma\left(\left(\boldsymbol{u}^{[j]}\right)^{m}\right)}
$$


where $\boldsymbol{u}^{[1]}, \ldots, \boldsymbol{u}^{[m]}$ are different sets of variables. The existence of such formulae helped motivate the new Abelian functions constructed in Section 6. For examples of using bases to calculate differential equations and addition formulae see [9, 16, 18, 19, 21].

While the $\wp$-functions may be defined algebraically using the curve, an alternative definition using the $\sigma$-function of the curve can be easier to work with. Recall that Weierstrass introduced an auxiliary function, $\sigma(u)$, in his theory which satisfies

$$
\wp(u)=-\frac{d^{2}}{d u^{2}} \log [\sigma(u)]
$$

The Weierstrass $\sigma$-function is usually defined as an infinite product over the periods, but it can also be expressed using the first Jacobi $\theta$-function, multiplied by a constant and exponential factor. This definition generalises naturally to give higher genus $\sigma$-functions as multivariate functions defined using the Riemann $\theta$-function Once the generalised $\sigma$-function is defined we may then consider generalised $\wp$-functions in analogy to the previous equation:

$$
\wp_{i j}(\boldsymbol{u})=-\frac{\partial^{2}}{\partial u_{i} \partial u_{j}} \log [\sigma(\boldsymbol{u})]
$$

Then, using the notation above, we see this is equivalent to the following definition in Hirota operators:

$$
\wp_{i j}=\left(\frac{-1}{2 \sigma^{2}}\right) S \circ \mathcal{D}_{i} \circ \mathcal{D}_{j}(\sigma \otimes \sigma),
$$

where $\mathcal{D}_{i}$ indicates that the differentiation in the Hirota operator is with respect to $u_{i}$. This led to the definition of so called $Q$-functions, where the operator is applied not twice but an even number of times, generating further Abelian functions with poles of order 2. See [9, 18, 20, 21] for examples of how such functions have been used to complete pole 2 bases. In this paper we provide the first proof that such functions are always Abelian.

These $Q$-functions led to the derivation of the generalised Hirota operators which are introduced in this paper. They allowed for the definition of more general class of Abelian functions which we call $\mathcal{R}$-functions. Infinitely many such functions can be defined with a given pole order, making them useful for the general basis problem.

The paper is divided into two parts. The first deals with the theory of the operators and the second the use of this theory in the construction of Abelian functions. While it is this application which is of primary interest in this paper, it is anticipated that the operators may have other uses, particularly in the world of integrable systems, and so we present their theory separately where possible. Section 2 reformulates the original Hirota theory into a form that more easily generalises, and proves some explicit results for the functions obtained by repeated application of the operators. Section 3 then defines the generalised operators and derives some of their properties. Sections 5 and 6 apply the operator theory to define infinite classes of Abelian functions, first with poles of order 2 and then poles of arbitrary order. Finally Section 7 uses these functions to construct new bases of Abelian functions for the two canonical curves of genus three.

\section{The Baker-Hirota operator}

We start by fixing some notation. Throughout the paper we are dealing with functions of $g$ variables. We drop the variables from our notation unless required. So for example,

$$
f=f(\boldsymbol{u})=f\left(u_{1}, u_{2}, \ldots, u_{g}\right) .
$$


When working with Abelian functions, $g$ will be the genus of some underlying algebraic curve. Until we come to the work in Section 7, none of the calculations in this document will depend on the particular curve or genus. In this section and the next the reader may assume we are just working in a $\mathbb{C}$-algebra of entire functions and their tensor products $\bigotimes_{\mathbb{C}}$.

Later we use subscripts on a function to indicate the application of operators, but for now we shall use subscripts to indicate differentiation with respect to one of the variables. For example,

$$
f_{i}=\frac{\partial}{\partial u_{i}} f\left(u_{1}, u_{2}, \ldots, u_{g}\right) .
$$

Superscripts in square brackets indicate a label, with other superscripts indicating the object raised to a power as normal.

Definition 2.1. We let $\mathcal{D}_{i}$ denote the Baker-Hirota operator. This acts on the tensor product of two functions as

$$
\mathcal{D}_{i}: f^{[1]} \otimes f^{[2]} \longrightarrow f_{i}^{[1]} \otimes f^{[2]}-f^{[1]} \otimes f_{i}^{[2]} .
$$

This operator is the key component in the famous Hirota derivative, discussed in the introduction. We refer to it as a Baker-Hirota operator since it was first used by Baker in [8].

We note that Baker-Hirota operators commute with each other,

$$
\mathcal{D}_{i} \circ \mathcal{D}_{j}\left(f^{[1]} \otimes f^{[2]}\right)=\mathcal{D}_{j} \circ \mathcal{D}_{i}\left(f^{[1]} \otimes f^{[2]}\right) .
$$

Definition 2.2. We let $S$ denote the $\mathbb{C}$-linear symmetrization operator which will send a tensor product to standard multiplication,

$$
S: f \otimes g \longrightarrow f g=g f .
$$

We are usually interested in applying the Baker-Hirota operator to a tensor product of the same object. We note that the symmetrization operator would annihilate the resulting sum after the application of a single operator,

$$
S \circ \mathcal{D}_{i}(f \otimes f)=S\left(f_{i} \otimes f-f \otimes f_{i}\right)=f_{i} f-f f_{i}=0 .
$$

However, when two operators are applied, there is a non-trivial result left after symmetrizing,

$$
S \circ \mathcal{D}_{i} \circ \mathcal{D}_{j}(f \otimes f)=S\left(\left(f_{i j} \otimes f-f_{i} \otimes f_{j}\right)-\left(f_{j} \otimes f_{i}-f \otimes f_{i j}\right)\right)=2\left(f f_{i j}-f_{i} f_{j}\right) .
$$

We will now consider the effect of multiple applications of a Baker-Hirota operator. We use $I_{n}=\left\{i_{1}, i_{2}, \ldots, i_{n}\right\}$ for the set of indices of the operators, assumed distinct for the moment. We introduce the multiple sum notation,

$$
\sum_{1 \leq j_{1}<j_{2}<\cdots<j_{m} \leq n}=\sum_{j_{1}=1}^{n} \sum_{j_{2}=j_{1}+1}^{n} \cdots \sum_{j_{m}=j_{m-1}+1}^{n} .
$$

Note that if there are no values of an index $j$ in the appropriate range then that sum does not run.

Lemma 2.3. For $n \geq 1$ we have

$$
\mathcal{D}_{i_{1}} \circ \cdots \circ \mathcal{D}_{i_{n}}(f \otimes g)=\sum_{m=0}^{n}(-1)^{m}\left(\sum_{1 \leq j_{1}<j_{2}<\cdots<j_{m} \leq n} f_{I_{n} \backslash\left\{i_{j_{1}}, i_{j_{2}}, \ldots, i_{j_{m}}\right\}} \otimes g_{i_{j_{1}} i_{j_{2}} \ldots i_{j_{m}}}\right) \text {. }
$$

When $m=0$ the entire inner sum is interpreted as a single term with the set $\left\{i_{j_{1}}, i_{j_{2}}, \ldots, i_{j_{m}}\right\}$ being the empty set, $\varnothing$. A function with an empty set of subscripts is just the function itself. 
We give the details of an inductive proof in Appendix A.

The lemma allows us to observe the following result, detailing when the symmetrization operator annihilates the result of Baker-Hirota operators applied to the same function.

Corollary 2.4. If $n$ is odd then $S \circ \mathcal{D}_{i_{1}} \circ \cdots \circ \mathcal{D}_{i_{n}}(f \otimes f)=0$.

Proof. We will show that when $n$ is odd, symmetrizing in equation (2.1) causes all terms to cancel. For example, the first term, $f_{I_{n}} \otimes f$, and the last term, $(-1)^{n} f \otimes f_{I_{n}}$ will cancel with each other.

First note that if $n$ is odd then the outer sum in (2.1) will have an even number of terms. We may consider these terms pairwise, when $m$ is $m_{1}=\left\lfloor\frac{n}{2}\right\rfloor-k$ and $m_{2}=\left\lceil\frac{n}{2}\right\rceil+k$, for some integer $k$. So each pair consists of the sums

$$
(-1)^{m_{1}} \sum_{1 \leq j_{1}<j_{2}<\cdots<j_{m} \leq n} f_{I_{n} \backslash\left\{i_{j_{1}}, i_{j_{2}}, \ldots, i_{j_{m_{1}}}\right\}} \otimes f_{i_{j_{1}}, i_{j_{2}}, \ldots, i_{j_{m_{1}}}}
$$

and

$$
(-1)^{m_{2}} \sum_{1 \leq j_{1}<j_{2}<\cdots<j_{m} \leq n} f_{I_{n} \backslash\left\{i_{j_{1}}, i_{j_{2}}, \ldots, i_{j_{m_{2}}}\right\}} \otimes f_{i_{j_{1}}, i_{j_{2}}, \ldots, i_{j_{m_{2}}}} .
$$

Now, $n$ is odd so the floor, \lfloor\rfloor and ceiling \lceil\rceil of $\frac{n}{2}$ will be of opposite relative parity, and hence so will $m_{1}$ and $m_{2}$ as they differ from the floor and ceiling by the same integer $k$.

Now since

$$
n=\left\lfloor\frac{n}{2}\right\rfloor+\left\lceil\frac{n}{2}\right\rceil,
$$

we have

$$
n-m_{1}=\left(\left\lfloor\frac{n}{2}\right\rfloor+\left\lceil\frac{n}{2}\right\rceil\right)-\left(\left\lfloor\frac{n}{2}\right\rfloor-k\right)=\left\lceil\frac{n}{2}\right\rceil+k=m_{2},
$$

and similarly $n-m_{2}=m_{1}$. Hence the partition of indices on either side of the tensor in the two terms are complementary. When we symmetrize they will give the same sum of terms, and so the differing signs outside the sums will cause them all to cancel.

Similar calculations will verify that the object is non-zero when $n$ is even.

We now introduce a multiplication on tensor products, which simply creates a tensor product of the same length by multiplying corresponding entries. For example,

$$
(a \otimes b)(c \otimes d)=(a c) \otimes(b d) .
$$

The operation is clearly symmetric;

$$
(a \otimes b)(c \otimes d)=(a c) \otimes(b d)=(c \otimes d)(a \otimes b) .
$$

Lemma 2.5. The Baker-Hirota operator satisfies a Leibniz rule. That is

$$
\mathcal{D}_{i}((a \otimes b)(c \otimes d))=(a \otimes b) \mathcal{D}_{i}(c \otimes d)+(c \otimes d) \mathcal{D}_{i}(a \otimes b) .
$$

Proof. By direct calculation we see that both sides of (2.2) are

$$
a_{i} c \otimes b d+a c_{i} \otimes b d-a c \otimes b_{i} d-a c \otimes b d_{i} .
$$


Corollary 2.6. The Baker-Hirota operators satisfy a general Leibniz rule,

$$
\mathcal{D}_{i_{1}} \circ \cdots \circ \mathcal{D}_{i_{n}}((a \otimes b)(c \otimes d))=\sum_{k=0}^{n} \sum_{\pi \in \Pi} \mathcal{D}_{\pi_{1}}(a \otimes b) \mathcal{D}_{\pi_{2}}(c \otimes d) .
$$

Here $\Pi$ is the set of ordered pairs of disjoint partitions, $\pi$, of the set of indices $I_{n}$ into subsets, $\pi_{1}$ and $\pi_{2}$ of length $n-k$ and $k$ respectively. The symbol $\mathcal{D}_{\pi_{i}}$ represents the concatenation of Baker-Hirota operators with indices the entries in $\pi_{i}$.

Proof. Recall that the standard product rule $(f g)^{\prime}=f^{\prime} g+f g^{\prime}$ leads by induction to the general Leibniz rule,

$$
(f g)^{(n)}=\sum_{k=0}^{n}\left(\begin{array}{l}
n \\
k
\end{array}\right) f^{(k)} g^{(n-k)},
$$

where $\left(\begin{array}{l}n \\ k\end{array}\right)$ are the binomial coefficients. This leads identically from Lemma 2.5 to the following result for a single Baker-Hirota operator,

$$
\mathcal{D}_{i}^{n}((a \otimes b)(c \otimes d))=\sum_{k=0}^{n}\left(\begin{array}{l}
n \\
k
\end{array}\right) \mathcal{D}_{i}^{k}(a \otimes b) \mathcal{D}_{i}^{n-k}(c \otimes d),
$$

where the superscripts on the operator mean it is applied multiple times. If instead we apply different Baker Hirota operators then we must consider all the different possibilities for $k$ applications of an operator in each step. This leads to the definition of $\Pi$ as in the corollary. The number of different possibilities is the number of ways of choosing $k$ from $n$, and so still $\left(\begin{array}{l}n \\ k\end{array}\right)$.

\section{Generalised Baker-Hirota operators}

In this section we consider a generalisation of the Baker-Hirota operators which maintains many of the properties from the previous section. Such operators will be classified with an order $m$, and will be multi-linear, acting on a tensor product of length $m$. We first introduce an operator to differentiate within such tensor products.

Definition 3.1. Define $\partial_{i}^{[j]}$ to be an operator which differentiates the $j$ th entry in a tensor product with respect to the variable $u_{i}$, i.e.

$$
\begin{aligned}
\partial_{i}^{[j]}\left(f^{[1]} \otimes \cdots \otimes f^{[j-1]} \otimes f^{[j]} \otimes f^{[j+1]} \otimes \cdots \otimes f^{[m]}\right) \\
\quad=f^{[1]} \otimes \cdots \otimes f^{[j-1]} \otimes f_{i}^{[j]} \otimes f^{[j+1]} \otimes \cdots \otimes f^{[m]} .
\end{aligned}
$$

We note that these operators commute with each other,

$$
\partial_{i_{1}}^{\left[j_{1}\right]} \partial_{i_{2}}^{\left[j_{2}\right]}=\partial_{i_{2}}^{\left[j_{2}\right]} \partial_{i_{1}}^{\left[j_{1}\right]} .
$$

For brevity we will often express these tensor products using a product notation, such as

$$
\bigotimes_{k=1}^{m} f^{[k]}=f^{[1]} \otimes \cdots \otimes f^{[m]}
$$

We now define the generalised operators. We note that these operators are similar to the (symmetric operators) proposed in [24]. However, the authors there considered only the trilinear cases $(m=3)$ in detail, and actually preferred to use a different non-symmetric basis that could be described as products of bilinear operators. Symmetric multilinear Hirota maps of the sort defined below were discussed in [3] in the context of algebraic covariant and invariant theory. 
Definition 3.2. Define $\mathcal{H}_{i}^{[m]}$ to be the $m$ th order generalised Baker-Hirota operator. This acts on the tensor product of $m$ functions as

$$
\mathcal{H}_{i}^{[m]}: \bigotimes_{k=1}^{m} f^{[k]} \longrightarrow \sum_{j=1}^{m} \zeta^{j-1} \partial_{i}^{[j]} \bigotimes_{k=1}^{m} f^{[k]}
$$

where $\zeta$ is a primitive $m$ th root of unity and $m \geq 2$.

We can take $\zeta$ to be any primitive $m$ th root of unity, but we may assume without loss of generality that

$$
\zeta=\exp \left(\frac{2 \pi i}{m}\right)
$$

Recall that

$$
1+\zeta+\zeta^{2}+\cdots+\zeta^{m-1}=0
$$

We note that the 2nd order generalised Baker-Hirota operator is exactly the original BakerHirota operator from Definition 2.1,

$$
\mathcal{H}_{i}^{[2]}=\mathcal{D}_{i} .
$$

For brevity we may write only $\mathcal{H}$-operator unless we mean to distinguish between the cases.

Since the $\partial_{i}^{[j]}$-operators commute with each other, it also clear that they commute with any $\mathcal{H}$-operator,

$$
\partial_{i_{1}}^{[j]} \circ \mathcal{H}_{i_{2}}^{[m]}=\mathcal{H}_{i_{2}}^{[m]} \circ \partial_{i_{1}}^{[j]} .
$$

From this we can conclude that the $\mathcal{H}$-operators of the same order commute with each other:

$$
\mathcal{H}_{i_{2}}^{[m]} \circ \mathcal{H}_{i_{1}}^{[m]}=\mathcal{H}_{i_{2}}^{[m]} \circ\left(\sum_{j_{1}=1}^{m} \zeta^{j_{1}-1} \partial_{i_{1}}^{\left[j_{1}\right]}\right)=\sum_{j_{1}=1}^{m} \zeta^{j_{1}-1} \partial_{i_{1}}^{\left[j_{1}\right]} \circ \mathcal{H}_{i_{2}}^{[m]}=\mathcal{H}_{i_{1}}^{[m]} \circ \mathcal{H}_{i_{2}}^{[m]} .
$$

We extend the symmetrization operator from Definition 2.2 to apply it to tensor products of arbitrary length,

$$
S: \bigotimes_{k=1}^{m} f^{[k]} \longrightarrow \prod_{k=1}^{m} f^{[k]}
$$

Again, we are usually interested in applying the generalised operators to a tensor product of the same object. As before, the symmetrization operator would annihilate the resulting sum after the application of a single operator:

$$
S \circ \mathcal{H}_{i}^{[m]}\left(\bigotimes_{k=1}^{m} f\right)=S\left(\sum_{j=1}^{m} \zeta^{j-1} \partial_{i}^{[j]} \bigotimes_{k=1}^{m} f^{[k]}\right)=\sum_{j=1}^{m} \zeta^{j-1} f^{m-1} f_{i}=0,
$$

where the final equality follows from equation (3.1). In this general case we find the symmetrization operator will also annihilate the result after two applications, so long as $m>2$. For example, consider applying the 3rd order operator twice:

$$
\mathcal{H}_{i_{1}}^{[3]} \bigotimes_{k=1}^{3} f=\zeta^{0} f_{i_{1}} \otimes f \otimes f+\zeta^{1} f \otimes f_{i_{1}} \otimes f+\zeta^{2} f \otimes f \otimes f_{i_{1}}
$$




$$
\begin{aligned}
\mathcal{H}_{i_{1}}^{[3]} \circ \mathcal{H}_{i_{2}}^{[3]} \bigotimes_{k=1}^{3} f= & \zeta^{0} f_{i_{1}, i_{2}} \otimes f \otimes f+\zeta^{1} f_{i_{1}} \otimes f_{i_{2}} \otimes f+\zeta^{2} f_{i_{1}} \otimes f \otimes f_{i_{2}} \\
& +\zeta^{1} f_{i_{2}} \otimes f_{i_{1}} \otimes f+\zeta^{2} f \otimes f_{i_{1}, i_{2}} \otimes f+\zeta^{3} f \otimes f_{i_{1}} \otimes f_{i_{2}} \\
& +\zeta^{2} f_{i_{2}} \otimes f \otimes f_{i_{1}}+\zeta^{3} f \otimes f_{i_{2}} \otimes f_{i_{1}}+\zeta^{4} f \otimes f \otimes f_{i_{1}, i_{2}} .
\end{aligned}
$$

So on symmetrizing we have

$$
S \circ \mathcal{H}_{i_{1}}^{[3]} \circ \mathcal{H}_{i_{2}}^{[3]} \bigotimes_{k=1}^{3} f=f_{i_{1} i_{2}} f^{2}\left(\zeta^{0}+\zeta^{2}+\zeta^{4}\right)+f_{i_{1}} f_{i_{2}} f\left(\zeta^{1}+\zeta^{2}+\zeta^{1}+\zeta^{3}+\zeta^{2}+\zeta^{3}\right) .
$$

Then since $\zeta^{3}=1$ we have

$$
S \circ \mathcal{H}_{i_{1}}^{[3]} \circ \mathcal{H}_{i_{2}}^{[3]} \bigotimes_{k=1}^{3} f=f_{i_{1} i_{2}} f^{2}\left(1+\zeta^{1}+\zeta^{2}\right)+2 f_{i_{1}} f_{i_{2}} f\left(1+\zeta^{1}+\zeta^{2}\right)=0 .
$$

To see what happens in general we start by considering the effect of multiple applications of the operators.

\subsection{Multiple applications of the generalised operators}

We are not able to use the multiple sum notation of Lemma 2.3. Instead we introduce some new notation, which may seem a little cumbersome at first, but is useful for proving the later results. This notation will be used throughout the rest of the paper.

Once again let $I_{n}=\left\{i_{1}, i_{2}, \ldots, i_{n}\right\}$ be a set of indices. Let $P_{n}^{m}$ be a set whose elements $\rho$ are sequences constructed from the partitions of the number $n$ of length at most $m$. If a partition has length less than $m$ then we extend it to length $m$ by adding zeros. We denote by $\rho_{1}, \ldots, \rho_{m}$ the parts of the partition in decreasing order and then we define $\rho$ to be the sequence of parts $\left[\rho_{1}, \ldots, \rho_{m}\right]$. Next we let $\Psi(\rho)$ be the set of permutations, $\psi$, of the sequence $\rho$. We denote by $\psi_{1}, \ldots, \psi_{m}$ the entries in a permuted sequence $\psi$. We define a function $z(\psi)$ which returns an integer according to

$$
z(\psi)=\sum_{k=1}^{m}(k-1)\left(\psi_{k}\right)
$$

Finally we let $\Pi(\psi)$ be the set of all disjoint partitions $\pi$ of the set of indices $I_{n}$ into $m$ subsets of lengths given by the sequence $\psi$. We denote the resulting subsets of such a $\pi$ by $\pi_{1}, \ldots, \pi_{m}$.

Lemma 3.3. For $n \geq 1$ we have

$$
\mathcal{H}_{i_{1}}^{[m]} \circ \cdots \circ \mathcal{H}_{i_{n}}^{[m]} \bigotimes_{k=1}^{m} f^{[k]}=\sum_{\rho \in P_{n}^{m}} \sum_{\psi \in \Psi(\rho)} \zeta^{z(\psi)} \sum_{\pi \in \Pi(\psi)} \bigotimes_{k=1}^{m} f_{\pi_{k}}^{[k]} .
$$

Proof. We do not need to give an inductive proof of Lemma 3.3 as it may be determined directly from a careful analysis of the resulting differential operator acting on the tensor product,

$$
\mathcal{H}_{i_{1}}^{[m]} \circ \cdots \circ \mathcal{H}_{i_{n}}^{[m]} \bigotimes_{k=1}^{m} f^{[k]}=\left(\prod_{k=1}^{n}\left(\sum_{j=1}^{m} \zeta^{j-1} \partial_{i_{k}}^{[j]}\right)\right) \bigotimes_{k=1}^{m} f^{[k]}
$$

The polynomial in $\partial$-operators would be fully symmetric if we had $\zeta=1$. We organise the terms as described in the lemma so that we collect together those with the same coefficient in $\zeta$ and we observe that this coefficient is then given using the function in equation (3.3). 
We note that the size of the set $\Pi(\psi)$ is the number of ways of splitting $n$ elements into $m$ groups of sizes $\psi_{1}, \ldots, \psi_{m}$. This is by definition a multinomial coefficient. Further, since $\psi$ is just a permutation of $\rho$, we have that

$$
|\Pi(\psi)|=|\Pi(\rho)|=\left(\begin{array}{c}
n \\
\rho_{1}, \ldots, \rho_{m}
\end{array}\right)=\frac{n !}{\rho_{1} ! \cdots \rho_{m} !} .
$$

We now give an example of the construction to demonstrate the notation. Consider the $m=3$, $n=2$ case that we looked at earlier. Here $I_{n}=\left\{i_{1}, i_{2}\right\}$ and so $P_{2}^{3}$ are the partitions of 2 with length at most 3 . There are two partitions, and we extend them each to length 3 to give

$$
\rho^{[1]}=[2,0,0] \quad \text { and } \quad \rho^{[2]}=[1,1,0] .
$$

First we consider $\rho^{[1]}$. There are three permutations of $[2,0,0]$ and so three entries in $\Psi\left(\rho^{[1]}\right)$;

$$
\psi^{[1]}=[2,0,0], \quad \psi^{[2]}=[0,2,0], \quad \text { and } \quad \psi^{[3]}=[0,0,2] .
$$

We see that

$$
z\left(\psi^{[1]}\right)=(0)(2)+(1)(0)+(2)(0)=0,
$$

and similarly $z\left(\psi^{[2]}\right)=2$ and $z\left(\psi^{[3]}\right)=4$. For each $\psi \in \Psi$ there is only one $\pi \in \Pi(\psi)$ since there is only one possible split of the indices into subsets of these lengths. We have

$$
\begin{aligned}
& \Pi\left(\psi^{[1]}\right)=\left\{\left[\left\{i_{1}, i_{2}\right\},\{\},\{\}\right]\right\}, \quad \Pi\left(\psi^{[2]}\right)=\left\{\left[\{\},\left\{i_{1}, i_{2}\right\},\{\}\right]\right\} \\
& \text { and } \Pi\left(\psi^{[3]}\right)=\left\{\left[\{\},\{\},\left\{i_{1}, i_{2}\right\}\right]\right\} .
\end{aligned}
$$

Hence $\rho^{[1]}$ contributes three terms to the sum;

$$
\zeta^{0} f_{i_{1}, i_{2}}^{[1]} \otimes f^{[2]} \otimes f^{[3]}+\zeta^{2} f^{[1]} \otimes f_{i_{1}, i_{2}}^{[2]} \otimes f^{[3]}+\zeta^{4} f^{[1]} \otimes f^{[2]} \otimes f_{i_{1}, i_{2}}^{[3]} .
$$

Now we consider $\rho^{[2]}$. There are also three permutations of $[1,1,0]$ and so again three entries in $\Psi\left(\rho^{[2]}\right)$;

$$
\psi^{[1]}=[1,1,0], \quad \psi^{[2]}=[1,0,1], \quad \text { and } \quad \psi^{[3]}=[0,1,1] .
$$

We calculate $z\left(\psi^{[1]}\right)=1, z\left(\psi^{[2]}\right)=2, z\left(\psi^{[3]}\right)=3$. In each of these case there are two possible partitions of the indices into sets of these lengths. For the first we have

$$
\Pi\left(\psi^{[1]}\right)=\left\{\left[\left\{i_{1}\right\},\left\{i_{2}\right\},\{\}\right],\left[\left\{i_{2}\right\},\left\{i_{1}\right\},\{\}\right]\right\} .
$$

This contributes the terms

$$
\zeta^{1}\left(f_{i_{1}}^{[1]} \otimes f_{i_{2}}^{[2]} \otimes f^{[3]}+f_{i_{2}}^{[1]} \otimes f_{i_{1}}^{[2]} \otimes f^{[3]}\right)
$$

to the sum. Similarly we have $\Pi\left(\psi^{[2]}\right)=\left\{\left[\left\{i_{1}\right\},\{\},\left\{i_{2}\right\}\right],\left[\left\{i_{2}\right\},\{\},\left\{i_{1}\right\}\right]\right\}$ contributing

$$
\zeta^{2}\left(f_{i_{1}}^{[1]} \otimes f^{[2]} \otimes f_{i_{2}}^{[3]}+f_{i_{2}}^{[1]} \otimes f^{[2]} \otimes f_{i_{1}}^{[3]}\right)
$$

and $\Pi\left(\psi^{[3]}\right)=\left\{\left[\{\},\left\{i_{1}\right\},\left\{i_{2}\right\}\right],\left[\{\},\left\{i_{2}\right\},\left\{i_{1}\right\}\right]\right\}$ contributing

$$
\zeta^{3}\left(f^{[1]} \otimes f_{i_{1}}^{[2]} \otimes f_{i_{2}}^{[3]}+f^{[1]} \otimes f_{i_{2}}^{[2]} \otimes f_{i_{1}}^{[3]}\right) .
$$

So we see we have found the nine terms expected from our calculation in equation (3.2). 


\subsection{Application on the tensor product of a function with itself}

We will now restrict to the case where the functions in the tensor product are taken to be the same, and consider the effect of the symmetrization operator. In this section we make use of the theory of symmetric functions. We gather together the necessary definitions and theory in Appendix B.

Lemma 3.4. Let $X=\left[X_{1}, \ldots, X_{m}\right]$ be given by $X_{k}=\zeta^{k-1}$. Then for $n \geq 1$ we have

$$
S \circ \mathcal{H}_{i_{1}}^{[m]} \circ \cdots \circ \mathcal{H}_{i_{n}}^{[m]} \bigotimes_{k=1}^{m} f=\sum_{\rho \in P_{n}^{m}} M_{\rho}(X) \sum_{\pi \in \Pi(\rho)}\left(\prod_{k=1}^{m} f_{\pi_{k}}\right) \text {, }
$$

where $M$ are the monomial symmetric functions (see Definition B.1).

Proof. We consider equation (3.4) from Lemma 3.3. Under the symmetrization operator $S$ the tensor product becomes a regular product, so

$$
S\left(\sum_{\pi \in \Pi(\psi)} \bigotimes_{k=1}^{m} f_{\pi_{k}}^{[k]}\right)=\sum_{\pi \in \Pi(\psi)} \prod_{k=1}^{m} f_{\pi_{k}}^{[k]}
$$

Now if all the functions $f^{[k]}=f$ then this sum over $\Pi(\psi)$ is the same for all $\psi \in \Psi(\rho)$. So we may instead denote it as a sum over $\Pi(\rho)$ and factor this entire object outside of the sum over $\Psi(\rho)$. We cannot discard the permutations $\psi$ entirely though, as the term $\zeta^{z(\psi)}$ still varies, even under symmetrization. We have

$$
S \circ \mathcal{H}_{i_{1}}^{[m]} \circ \cdots \circ \mathcal{H}_{i_{n}}^{[m]} \bigotimes_{k=1}^{m} f=\sum_{\rho \in P_{n}^{m}}\left(\sum_{\pi \in \Pi(\rho)}\left(\prod_{k=1}^{m} f_{\pi_{k}}\right)\right)\left(\sum_{\psi \in \Psi(\rho)} \zeta^{z(\psi)}\right) .
$$

We further analyse the sum in the final factor. We have

$$
\sum_{\psi \in \Psi(\rho)} \zeta^{z(\psi)}=\sum_{\psi \in \Psi(\rho)} \prod_{k=1}^{m}\left(\zeta^{k-1}\right)^{\psi_{k}}
$$

If we introduce the dummy variables $X_{k}=\zeta^{k-1}$ then we have that

$$
\sum_{\psi \in \Psi(\rho)} \zeta^{z(\psi)}=\sum_{\psi \in \Psi(\rho)} \prod_{k=1}^{m} X_{k}^{\psi_{k}}=M_{\rho}\left(X_{1}, \ldots, X_{m}\right)
$$

from Definition B.1. Hence the final factor is identified as $M_{\rho}(X)$ as described in the lemma.

Denote the power sum symmetric functions by $p_{k}$ (see Definition B.2). When evaluated on $X$ as in Lemma 3.4 we find (see Lemma B.3) that

$$
p_{k}= \begin{cases}m & \text { if } m \mid k \\ 0 & \text { otherwise }\end{cases}
$$

By considering expressions in the power sum basis we can determine when multiple applications of the $\mathcal{H}$-operators map to zero.

Corollary 3.5. If $m \nmid n$ then

$$
S \circ \mathcal{H}_{i_{1}}^{[m]} \circ \cdots \circ \mathcal{H}_{i_{n}}^{[m]} \bigotimes_{k=1}^{m} f=0 .
$$


Proof. Every monomial symmetric function is homogeneous in degree. For those appearing in Lemma 3.4, the degree is always $n$. We may think of the degree as a weight. Since each power sum $p_{k}$ has degree $k$, the expression for $M_{\rho}$ as a polynomial in $p_{1}, \ldots, p_{m}$ will only involve terms where the subscripts of the power sums add to $n$. Hence if $m \nmid n$ then the expression cannot contain any terms with $p_{m}$ as the only variable. However, it is only these terms that do not evaluate to zero with the choice of variables $X_{k}=\zeta^{k-1}$ by Lemma B.3.

So if $m \mid n$ then every term in the right hand side of equation (3.5) is zero.

Corollary 3.6. If $m \mid n$ then $S \circ \mathcal{H}_{i_{1}}^{[m]} \circ \cdots \circ \mathcal{H}_{i_{n}}^{[m]} \bigotimes_{k=1}^{m} f$ is not identically zero.

Proof. Consider the expression in Lemma 3.4. One of the terms in equation (3.5) will correspond the partition on $n$ with $\rho=[n, 0, \ldots, 0]$. But for such a partition $M_{\rho}=p_{n}$ and by Lemma B.3 we know that this power sum is not zero for $m \mid n$. So one of the terms in the expression is a non-zero constant times a monomial in derivatives of $f$ which does not occur in any of the other terms. Hence the expression cannot be identically zero.

We can go further and give a formula for the value of the $M_{\rho}$ in the case where $n \mid m$. To do this we use a procedure of Doubilet from [15] to express the monomial symmetric functions in power sums with coefficients given by Möbius functions, $\mu(\pi)$. We give the full details in Appendix B with the key formula stated in equation (B.2).

Corollary 3.7. The augmented monomial symmetric functions on $X=\left[1, \zeta, \ldots, \zeta^{m-1}\right]$ are

$$
\hat{M}_{\rho}=\sum_{\pi \in \Pi^{\prime}} \mu(\pi) m^{\ell}
$$

where $\ell$ is the number of subsets in $\pi$ and $\Pi^{\prime}$ contains only those set partitions $\pi \in \Pi$ such that all parts of $\boldsymbol{\nu}(\boldsymbol{\rho}, \boldsymbol{\pi})$ are multiples of $m$.

Proof. From Lemma B.3 we know that when evaluating equation (B.2) on the variables $X_{k}=\zeta^{k-1}$, only those terms such that all parts of $\boldsymbol{\nu}(\boldsymbol{\rho}, \boldsymbol{\pi})$ are multiples of $m$ will be nonzero. In these cases each $p_{i}$ is $m$ and so each Möbius function has a coefficient of $m$ to the power of the length of $\boldsymbol{\nu}(\boldsymbol{\rho}, \boldsymbol{\pi})$.

The monomial symmetric functions involved in Lemma 3.4 will evaluate to the integers for the augmented functions from the corollary above, divided by the constants from equation (B.1). However, we observe that when $m \mid n$ then $S \circ \mathcal{H}_{i_{1}}^{[m]} \circ \cdots \circ \mathcal{H}_{i_{n}}^{[m]} \bigotimes_{k=1}^{m} f$ also has integer coefficients as a polynomial in the derivatives of $f$. In fact, these integer coefficients all have a common factor of $m$.

\subsection{Leibniz rule}

We recall the multiplication operation we defined for tensor products, which multiplies corresponding entries in tensor products of the same length. We use it again here to show that the $\mathcal{H}$-operators all satisfy Leibniz properties.

Lemma 3.8. An $m$ th order $\mathcal{H}$-operator satisfies a product rule. That is

$$
\begin{aligned}
\mathcal{H}_{i}^{[m]} & \left(\left(\bigotimes_{k=1}^{m} f^{[k]}\right)\left(\bigotimes_{k=1}^{m} g^{[k]}\right)\right) \\
& =\left(\bigotimes_{k=1}^{m} f^{[k]}\right) \mathcal{H}_{i}^{[m]}\left(\bigotimes_{k=1}^{m} g^{[k]}\right)+\left(\bigotimes_{k=1}^{m} g^{[k]}\right) \mathcal{H}_{i}^{[m]}\left(\bigotimes_{k=1}^{m} f^{[k]}\right) .
\end{aligned}
$$


Proof. Using the definitions we have

$$
\begin{aligned}
& \operatorname{LHS}(3.6)=\sum_{j=1}^{m} \zeta^{j-1} \partial_{i}^{[j]} \bigotimes_{k=1}^{m} f^{[k]} g^{[k]}, \\
& \operatorname{RHS}(3.6)=\sum_{j=1}^{m} \zeta^{j-1}\left(\left(\bigotimes_{k=1}^{m} f^{[k]}\right)\left(\partial_{i}^{[j]} \bigotimes_{k=1}^{m} g^{[k]}\right)+\left(\bigotimes_{k=1}^{m} g^{[k]}\right)\left(\partial_{i}^{[j]} \bigotimes_{k=1}^{m} f^{[k]}\right)\right) .
\end{aligned}
$$

Comparing the $j$ th term in each sum shows them to be equal, (and hence that the operator $\partial_{i}^{[j]}$ satisfies its own product rule).

Corollary 3.9. The $\mathcal{H}$-operators satisfy a general Leibniz rule,

$$
\mathcal{H}_{i_{1}}^{[m]} \circ \cdots \circ \mathcal{H}_{i_{n}}^{[m]}\left(\bigotimes_{k=1}^{m} f^{[k]}\right)\left(\bigotimes_{k=1}^{m} g^{[k]}\right)=\sum_{\ell=0}^{n} \sum_{\pi \in \Pi} \mathcal{H}_{\pi_{1}}^{[m]}\left(\bigotimes_{k=1}^{m} f^{[k]}\right) \mathcal{H}_{\pi_{2}}^{[m]}\left(\bigotimes_{k=1}^{m} g^{[k]}\right) .
$$

Here $\Pi$ is again the set of disjoint partitions, $\pi$, of the set of indices $I_{n}$ into two subsets, $\pi_{1}$ and $\pi_{2}$, of length $n-\ell$ and $\ell$ respectively. The symbol $\mathcal{H}_{\pi_{i}}^{[m]}$ represents the concatenation of $\mathcal{H}$-operators of the same order with indices the entries in $\pi_{i}$.

Proof. This follows the proof of the original Leibniz rule and the proof of Corollary 2.6.

We note that as in Corollary 2.6, the size of $\Pi$ is still the binomial coefficient $\left(\begin{array}{l}n \\ \ell\end{array}\right)$.

\section{Abelian functions associated with algebraic curves}

The first generalisation of the elliptic functions to hyperelliptic functions was pioneered by Klein and Baker as described in Baker's classic texts [6,7]. They defined multivariate $\wp$-functions as discussed in Section 1. Hence these generalised functions are sometimes called Kleinian. The more general definitions presented on this section are primarily from the work of Buchstaber, Enolskii and Leykin in [11] and the further developments in [17] and [33].

The periodicity conditions of all the higher genus functions are defined using the period lattice of an underlying algebraic curve. We use the higher genus $\sigma$-function of the curve to define them. We give explicit definitions relating to the class of $(n, s)$-curves, defined below. This class is special as the curves all have a branch point at infinity. The restriction to this class is so that we can make use of the well developed theory of series expansions for the functions associated to such curves to check the linear independence of functions.

However, we note that one can define Abelian functions associated to curves outside this class. One of the authors has pioneered a so called equivariant approach to the functions in [4], where the curve and functions all transform under an $\mathfrak{s l}_{2}$ action. Another recent development is in [28] where the authors define $\sigma$-functions associated to Riemann surfaces without making any realisation of the surface as an algebraic equation. The definitions and results in Sections 5 and 6 for Abelian functions defined using $\mathcal{H}$-operators should be applicable in these alternative theories. It is only in the calculation of the explicit bases in Section 7, that we make use of the $(n, s)$-theory.

Definition 4.1. For two coprime integers $(n, s)$ with $s>n$ we define an $(n, s)$-curve, denoted $C$, as an algebraic curve defined by $f(x, y)=0$, where

$$
f(x, y)=y^{n}+q_{1}(x) y^{n-1}+q_{2}(x) y^{n-2}+\cdots+q_{n-1}(x) y-q_{n}(x) .
$$


Here $x, y$ are complex variables and $q_{j}(x)$ are polynomials in $x$ of degree (at most) $\lfloor j s / n\rfloor$. We define a simple subclass of the curves by setting $q_{j}(x)=0$ for $0 \leq j \leq n-1$. These simpler curves are defined using curve parameters $\lambda_{j}$ as

$$
f(x, y)=y^{n}-\left(x^{s}+\lambda_{s-1} x^{s-1}+\cdots+\lambda_{1} x+\lambda_{0}\right)
$$

and are called cyclic $(n, s)$-curves. In the literature the word 'cyclic' is sometimes replaced by 'strictly' or 'purely $n$-gonal'. Note that we also have curve parameters for the general $(n, s)$ curves, contained within the $q_{j}(x)$.

Restricting to the cyclic classes results in much easier computations, but it not usually necessary for theoretical reasons. However, the cyclic curves do possess extra symmetry which manifests itself in the fact that the associated functions satisfy a wider set of addition formulae.

If the curve $C$ is a non-singular model of $X$ then the genus is given by $g=\frac{1}{2}(n-1)(s-1)$. The associated functions will be multivariate with $g$ variables, $\boldsymbol{u}=\left(u_{1}, \ldots, u_{g}\right)$. For example, a $(2,3)$-curve would have genus one and is indeed an elliptic curve. The associated Weierstrass $\sigma$ and $\wp$-functions depend upon a single complex variable $u$.

\subsection{Defining the functions}

We first describe how the period lattice associated to the curve may be constructed. We start by choosing a basis for the space of differential forms of the first kind; the differential 1-forms which are holomorphic on the curve, $C$. There is a standard procedure to construct this basis for an $(n, s)$-curve (see for example [33]). In general the basis is given by $h_{i} d x / f_{y}, i=1, \ldots, g$ where the $h_{i}$ are monomials in $(x, y)$ whose structure may be predicted by the Weierstrass gap sequence, although other normalisations are sometimes used.

We next choose a symplectic basis in $H_{1}(C, \mathbb{Z})$ of cycles (closed paths) upon the compact Riemann surface defined by $C$. We denote these by $\left\{\alpha_{1}, \ldots, \alpha_{g}, \beta_{1}, \ldots, \beta_{g}\right\}$. We ensure the cycles have intersection numbers

$$
\alpha_{i} \alpha_{j}=0, \quad \beta_{i} \beta_{j}=0, \quad \alpha_{i} \beta_{j}=\delta_{i j}= \begin{cases}1 & \text { if } i=j \\ 0 & \text { if } i \neq j\end{cases}
$$

The choice of these cycles is not unique, but the functions will be independent of the choice.

We introduce $\boldsymbol{d} \boldsymbol{r}$ as a basis of differentials of the second kind. These are meromorphic differentials on $C$ which have their only pole at $\infty$. This basis is usually derived alongside the fundamental differential of the second kind. Rather than repeat the full details here we refer the reader to [6] for the general theory and [10] which gives a detailed example construction.

We can now define the standard period matrices associated to the curve as

$$
\begin{aligned}
\omega^{\prime} & =\left(\oint_{\alpha_{k}} d u_{\ell}\right)_{k, \ell=1, \ldots, g}, & \omega^{\prime \prime} & =\left(\oint_{\beta_{k}} d u_{\ell}\right)_{k, \ell=1, \ldots, g}, \\
\eta^{\prime} & =\left(\oint_{\alpha_{k}} d r_{\ell}\right)_{k, \ell=1, \ldots, g}, & \eta^{\prime \prime} & =\left(\oint_{\beta_{k}} d r_{\ell}\right)_{k, \ell=1, \ldots, g}
\end{aligned}
$$

and define the period lattice $\Lambda$ formed from $\omega^{\prime}, \omega^{\prime \prime}$ by

$$
\Lambda=\left\{\omega^{\prime} \boldsymbol{m}+\omega^{\prime \prime} \boldsymbol{n}, \boldsymbol{m}, \boldsymbol{n} \in \mathbb{Z}^{g}\right\} .
$$

This is a lattice in the space $\mathbb{C}^{g}$. The Jacobian variety of $C$ is presented by $\mathbb{C}^{g} / \Lambda$, and is denoted by $J$. We define $\kappa$ as the modulo $\Lambda$ map,

$$
\kappa: \mathbb{C}^{g} \rightarrow J
$$


For $k=1,2, \ldots$ define $\mathfrak{A}_{\mathfrak{k}}$, the Abel map from the $k$-th symmetric product $\operatorname{Sym}^{k}(C)$ of $C$ to $J$ by

$$
\begin{aligned}
\mathfrak{A}_{\mathfrak{k}}: \operatorname{Sym}^{k}(C) & \rightarrow J, \\
& \left(P_{1}, \ldots, P_{k}\right) \mapsto\left(\int_{\infty}^{P_{1}} \boldsymbol{d u}+\cdots+\int_{\infty}^{P_{k}} \boldsymbol{d u}\right) \quad(\bmod \Lambda),
\end{aligned}
$$

where the $P_{i}$ are again points upon $C$. Denote the image of the $k$-th Abel map by $W^{[k]}$ and define the $k$-th standard theta subset (sometimes referred to as the $k$-th strata) by

$$
\Theta^{[k]}=W^{[k]} \cup[-1] W^{[k]},
$$

where $[-1]$ means that

$$
[-1]\left(u_{1}, \ldots, u_{g}\right)=\left(-u_{1}, \ldots,-u_{g}\right) .
$$

We are considering functions that are periodic with respect to the lattice $\Lambda$.

Definition 4.2. Let $\mathfrak{M}(\boldsymbol{u})$ be a meromorphic function of $\boldsymbol{u} \in \mathbb{C}^{g}$. Then $\mathfrak{M}$ is a standard Abelian function associated with $C$ if it has poles only along $\kappa^{-1}\left(\Theta^{[g-1]}\right)$ and satisfies, for all $\boldsymbol{\ell} \in \Lambda$,

$$
\mathfrak{M}(\boldsymbol{u}+\boldsymbol{\ell})=\mathfrak{M}(\boldsymbol{u})
$$

All the Abelian functions in this paper are defined using the $\sigma$-function of the curve. While not an Abelian function itself, it does satisfy a quasi-periodicity property. Let $\boldsymbol{\delta}=\omega^{\prime} \boldsymbol{\delta}^{\prime}+\omega^{\prime \prime} \boldsymbol{\delta}^{\prime \prime}$ be the Riemann constant with base point $\infty$. Then $[\boldsymbol{\delta}]$ is the theta characteristic representing the Riemann constant for the curve $C$ with respect to the base point $\infty$ and generators $\left\{\alpha_{j}, \beta_{j}\right\}$ of $H_{1}(C, \mathbb{Z})$. (See for example [11, pp. 23-24].)

Definition 4.3. The Kleinian $\sigma$-function associated to a general $(n, s)$-curve is defined using a multivariate $\theta$-function with characteristic $\boldsymbol{\delta}$ as

$$
\begin{aligned}
\sigma(\boldsymbol{u})= & c \exp \left(\frac{1}{2} \boldsymbol{u} \eta^{\prime}\left(\omega^{\prime}\right)^{-1} \boldsymbol{u}^{T}\right) \theta[\boldsymbol{\delta}]\left(\left(\omega^{\prime}\right)^{-1} \boldsymbol{u}^{T} \mid\left(\omega^{\prime}\right)^{-1} \omega^{\prime \prime}\right)=c \exp \left(\frac{1}{2} \boldsymbol{u} \eta^{\prime}\left(\omega^{\prime}\right)^{-1} \boldsymbol{u}^{T}\right) \\
& \times \sum_{\boldsymbol{m} \in \mathbb{Z}^{g}} \exp \left[2 \pi i\left\{\frac{1}{2}\left(\boldsymbol{m}+\boldsymbol{\delta}^{\prime}\right)^{T}\left(\omega^{\prime}\right)^{-1} \omega^{\prime \prime}\left(\boldsymbol{m}+\boldsymbol{\delta}^{\prime}\right)+\left(\boldsymbol{m}+\boldsymbol{\delta}^{\prime}\right)^{T}\left(\left(\omega^{\prime}\right)^{-1} \boldsymbol{u}^{T}+\boldsymbol{\delta}^{\prime \prime}\right)\right\}\right] .
\end{aligned}
$$

The constant $c$ is dependent upon the curve parameters and the basis of cycles and is fixed later, following Lemma 4.8 .

We now summarise the key properties of the $\sigma$-function. See [11] or [33] for the construction of the $\sigma$-function to satisfy these properties. For any point $\boldsymbol{u} \in \mathbb{C}^{g}$ we denote by $\boldsymbol{u}^{\prime}$ and $\boldsymbol{u}^{\prime \prime}$ the vectors in $\mathbb{R}^{g}$ such that

$$
\boldsymbol{u}=\omega^{\prime} \boldsymbol{u}^{\prime}+\omega^{\prime \prime} \boldsymbol{u}^{\prime \prime}
$$

Therefore a point $\ell \in \Lambda$ is written as

$$
\ell=\omega^{\prime} \ell^{\prime}+\omega^{\prime \prime} \ell^{\prime \prime} \in \Lambda, \quad \ell^{\prime}, \ell^{\prime \prime} \in \mathbb{Z}^{g} .
$$

For $\boldsymbol{u}, \boldsymbol{v} \in \mathbb{C}^{g}$ and $\boldsymbol{\ell} \in \Lambda$, define $L(\boldsymbol{u}, \boldsymbol{v})$ and $\chi(\boldsymbol{\ell})$ as follows:

$$
\begin{aligned}
& \hat{L}(\boldsymbol{u}, \boldsymbol{v})=\boldsymbol{u}^{T}\left(\eta^{\prime} \boldsymbol{v}^{\prime}+\eta^{\prime \prime} \boldsymbol{v}^{\prime \prime}\right), \\
& \chi(\boldsymbol{\ell})=\exp \left[2 \pi \mathrm{i}\left\{\left(\boldsymbol{\ell}^{\prime}\right)^{T} \delta^{\prime \prime}-\left(\boldsymbol{\ell}^{\prime \prime}\right)^{T} \delta^{\prime}+\frac{1}{2}\left(\boldsymbol{\ell}^{\prime}\right)^{T} \boldsymbol{\ell}^{\prime \prime}\right\}\right] .
\end{aligned}
$$


Lemma 4.4. Consider the $\sigma$-function associated to an $(n, s)$-curve.

- It is an entire function on $\mathbb{C}^{g}$.

- It has zeros of order one along the set $\kappa^{-1}\left(\Theta^{[g-1]}\right)$, commonly called the $\Theta$-divisor. Further, we have $\sigma(\boldsymbol{u}) \neq 0$ outside the set.

- For all $\boldsymbol{u} \in \mathbb{C}^{g}, \boldsymbol{\ell} \in \Lambda$ the function has the quasi-periodicity property:

$$
\sigma(\boldsymbol{u}+\boldsymbol{\ell})=\chi(\ell) \exp \left[\hat{L}\left(\boldsymbol{u}+\frac{\boldsymbol{\ell}}{2}, \boldsymbol{\ell}\right)\right] \sigma(\boldsymbol{u})
$$

We let $L=\hat{L}\left(\boldsymbol{u}+\frac{\ell}{2}\right)$ and $h=\chi e^{L}$ denote the extra factor introduced. So $\sigma(\boldsymbol{u}+\ell)=h \sigma(\boldsymbol{u})$.

- It has definite parity given by

$$
\sigma(-\boldsymbol{u})=(-1)^{\frac{1}{24}\left(n^{2}-1\right)\left(s^{2}-1\right)} \sigma(\boldsymbol{u}) .
$$

Proof. The function is clearly entire from the definition, while the quasi-periodicity and the zeros are classical results (see [6]), that are fundamental to the definition of the function. They both follow from the properties of the multivariate $\theta$-function. The parity property is given by Proposition 4(iv) in [33].

We can now define $\wp$-functions using an analogy of the relation between $\wp$ and $\sigma$ in the elliptic case.

Definition 4.5. Define $m$-index Kleinian $\wp$-functions for $m \geq 2$ by

$$
\wp_{i_{1}, i_{2}, \ldots, i_{m}}(\boldsymbol{u})=-\frac{\partial}{\partial u_{i_{1}}} \frac{\partial}{\partial u_{i_{2}}} \cdots \frac{\partial}{\partial u_{i_{m}}} \log [\sigma(\boldsymbol{u})]
$$

where $i_{1} \leq \cdots \leq i_{m} \in\{1, \ldots, g\}$.

The $m$-index $\wp$-functions are meromorphic with poles of order $m$ when $\sigma(\boldsymbol{u})=0$. We can check that they satisfy equation (4.3) and hence they are Abelian by substituting equation (4.4) directly into their definition. The $m$-index $\wp$-functions have definite parity with respect to the change of variables $\boldsymbol{u} \rightarrow[-1] \boldsymbol{u}$. This is independent of the underlying curve, with the functions odd if $m$ is odd and even if $m$ is even. The ordering of the indices is irrelevant and so for simplicity we always order in ascending value.

The new Abelian functions introduce in Sections 5 and 6 are defined using the operators applied to tensor products of functions. We define an operator for checking their periodicity.

Definition 4.6. Given the lattice $\Lambda$, define a corresponding lattice operator by

$$
\Lambda_{\ell}: \boldsymbol{u} \mapsto \boldsymbol{u}+\ell
$$

for $\ell$ some arbitrary point in the lattice. So $\Lambda_{\ell}(\sigma)=\sigma(\boldsymbol{u}+\ell)=h \sigma$ for example. In a slight abuse of notation we also use $\Lambda_{\ell}$ for the operator acting on a tensor product,

$$
\Lambda_{\ell} \bigotimes_{i=1}^{n} f^{[i]}=\bigotimes_{i=1}^{n} \Lambda_{\ell} f^{[i]}
$$

Lemma 4.7. The operator $\Lambda_{\ell}$ commutes with $S, \mathcal{D}_{i}$ and $\mathcal{H}_{i}^{[m]}$.

The proofs are all simple by direct calculation. 


\subsection{Weights and expansions}

For a given $(n, s)$-curve we can define a set of weights, denoted by wt and often referred to as the Sato weights. We start by setting $\mathrm{wt}(x)=-n, \operatorname{wt}(y)=-s$ and then choose the weights of the curve parameters to be such that the curve equation is homogeneous. We see that for cyclic curves this imposes wt $\left(\lambda_{j}\right)=-n(s-j)$. The Abel map $\mathfrak{A}_{1}$ gives an embedding of the curve $C$ upon which we can define $\xi=x^{-\frac{1}{n}}$ as the local parameter at the origin, $\mathfrak{A}_{1}(\infty)$. We can then express $y$ and the basis of differentials using $\xi$ and integrate to give series expansions for $\boldsymbol{u}$. We can check the weights of $\boldsymbol{u}$ from these expansions and see that they are prescribed by the Weierstrass gap sequence.

By considering Definition 4.5 we see that the weight of the $\wp$-functions is the negative of the sum of the weights of the variables indicated by the indices, irrespective of what the weight of $\sigma(\boldsymbol{u})$ is. We note that curves of the same genus will, notationally, have the same $\wp$-functions, but may exhibit different behavior as indicated by the different weights of the variables and functions. We will discuss the weight of the $\sigma$-function below. All other functions discussed are constructed from $\sigma$ or $\wp$-functions and their weights follow accordingly. We can show that all the equations in the theory are homogeneous in these weights, with a more detailed discussion of this available, for example, in [21].

We can construct a series expansion of the $\sigma$-function about the origin, as described below.

Lemma 4.8. The Taylor series expansion of $\sigma(\boldsymbol{u})$ about the origin may be written as

$$
\sigma(\boldsymbol{u})=K S W_{n, s}(\boldsymbol{u})+\sum_{k=0}^{\infty} C_{k}(\boldsymbol{u}) .
$$

Here $K$ is a constant, $S W_{n, s}$ the Schur-Weierstrass polynomial generated by $(n, s)$ and each $C_{k}$ a finite, polynomial composed of products of monomials in $\boldsymbol{u}$ of weight $k$ multiplied by monomials in the curve parameters of weight $-(w t(\sigma)-k)$.

Proof. We refer the reader to [33] for a proof of the relationship between the $\sigma$-function and the Schur-Weierstrass polynomials and note that this was first discussed in [12]. We see that the remainder of the expansion must depend on the curve parameters and split it up into the different $C_{k}$ according to the weight split on terms between the parameters and $\boldsymbol{u}$. We can see that each $C_{k}$ is finite since the number of possible terms with the prescribed weight properties is finite. In fact, by considering the possible weights of the curve coefficients we see that the index $k$ in the sum will, in the cyclic case, actually increase in multiples of $n$.

The Schur-Weierstrass polynomials are Schur polynomials generated by a Weierstrass partition, derived in turn from the Weierstrass gap sequence for $(n, s)$. See [33] and [12] for more details on these polynomials. This connection with the Schur-Weierstrass polynomials allows us to determine the weight of the $\sigma$-function as

$$
\operatorname{wt}(\sigma)=(1 / 24)\left(n^{2}-1\right)\left(s^{2}-1\right) .
$$

In Definition 4.3 we fix $c$ to be the value that makes the constant $K=1$ in the above lemma. Some other authors working in this area may use a different constant and in general these choices are not equivalent. However, the constant can be seen to cancel in the definition of all Abelian functions, leaving results between the functions independent of $c$. Note that this choice of $c$ ensures that the Kleinian $\sigma$-function matches the Weierstrass $\sigma$-function when the $(n, s)$-curve is chosen to be the classic elliptic curve.

The expansion can be constructed by considering each $C_{k}$ in turn, identifying the possible terms, forming a series with unidentified coefficients and then determining the coefficients 
by ensuring the expansions satisfy known properties of the $\sigma$-function. For example, using its vanishing properties or the identities between the associated Abelian functions. Large expansions of this type were first introduced in [10], which used the generalised $\sigma$-function to construct explicit reductions of the Benney equations. Since then they have been an integral tool in the investigation of Abelian functions. Recently computational techniques based on the weight properties have been used to derive much larger expansions and we refer the reader to [21] and [20] for a more detailed discussion of the constructions.

Such expansions are possible for the general $(n, s)$-curves, but the calculations involved are far simpler for the cyclic cases. In this paper such expansions are used only to check linear independence in the examples of Section 7. The number of $C_{k}$ required to do this will depend on the weight of the functions in question. The expansion will need to go far enough to give the monomials in curve coefficients that may be present in any identity between functions.

\subsection{Bases of Abelian functions}

We can classify the Abelian functions according to their pole structure. We denote by $\Gamma(m)$ the vector space of Abelian functions defined upon $J$ which have poles of order at most $m$, occurring only on $\kappa^{-1}\left(\Theta^{[g-1]}\right)$, where the $\theta$ and $\sigma$-functions have their zeros and the Abelian functions their poles. This is usually called the $\Theta$-divisor.

A key problem is the generation of bases for these vector spaces. Note that the dimension of the space $\Gamma(m)$ is $m^{g}$ by the Riemann-Roch theorem for Abelian varieties (see for example [29]). The first step in constructing a basis is to include the entries in the preceding basis for $\Gamma(m-1)$. Subsequently only functions with poles of order exactly $m$ need to be sought. The Kleinian $\wp-$ functions are natural candidates and indeed are sufficient to solve the problem in the elliptic case. Here $g=1$ and so only one new function is required at each stage, which can be filled by the repeated derivatives of the Weierstrass $\wp$-functions.

However, if $g>1$ then new classes of functions are required to complete the bases. For example, in the genus 2 case we find that when considering $\Gamma(3)$ we need an additional entry after including all possible $\wp$-functions. The function

$$
\Delta=\wp_{11} \wp_{22}-\wp_{12}^{2}
$$

is usually taken to fill this hole. The individual terms in $\Delta$ have poles of order 4 , but when taken together they cancel to leave poles of order 3 as can easily be checked using Definition 4.5. We then proceed to consider $\Gamma(4)$ and find that two new functions are needed after the inclusion of the 4-index $\wp$-functions. The two derivatives of $\Delta$ can play this role. Naturally the derivatives of the $\Delta$ have poles of order four but we must also ensure that they are linearly independent of the other functions. This can be checked trivially by noting that they are of a different weight to both each other and all the other elements of the basis.

It is simple to check that when considering higher values of $m$, the preceding basis and its unique derivatives always give the required number of functions for a new basis, and that they are all of unique weight and so linearly independent. Hence the general basis is as described in Table 1 , where $\{\cdot\}$ is indicating all functions of this form.

The genus 1 and 2 cases, in which we get to a stage where new bases are calculated from the old ones and their derivatives, are special. They fall into the class where the theta divisor is nonsingular and the $\mathcal{D}$-module structure of such cases is discussed in [13]. For $g>2$ hyperelliptic and $g>3$ non-hyperelliptic curves this is not the case and so new methods are required to derive bases.

One approach to the problem is to define new functions by matching poles in algebraic combinations of $\wp$-functions so that they cancel, analogous to $\Delta$ above. This method was applied successfully to derive bases for various vector spaces in [19] and [16]. However, this 
Table 1. Table of bases for Abelian functions associated with a genus two curve.

\begin{tabular}{l|cl}
\hline Space & Dimension & Basis \\
\hline$\Gamma(0)$ & 1 & $\{1\}$ \\
$\Gamma(1)$ & 1 & $\{1\}$ \\
$\Gamma(2)$ & 4 & $\left\{1, \wp_{11}, \wp_{12}, \wp_{22}\right\}$ \\
$\Gamma(3)$ & 9 & $\left\{1, \wp_{11}, \wp_{12}, \wp_{22}, \wp_{111}, \wp_{112}, \wp_{122}, \wp_{222}, \Delta\right\}$ \\
$\Gamma(4)$ & 16 & $\left\{1, \ldots, \Delta, \wp_{1111}, \ldots, \wp_{2222}, \partial_{1} \Delta, \partial_{2} \Delta\right\}$ \\
$\vdots$ & $\vdots$ & \\
$\Gamma(m)$ & $m^{2}$ & $\{$ basis for $\Gamma(m-1)\} \cup\left\{\left\{\wp_{i_{1} \ldots i_{m}}\right\},\left\{\partial_{i_{1}} \cdots \partial_{i_{m-2}} \Delta\right\}\right\}$
\end{tabular}

approach does not generalise easily, which led to the development of the new classes of Abelian functions in Sections 5 and 6.

\section{$5 \quad Q$-functions}

In this section we study the $Q$-functions, defined using the original Baker-Hirota operator, proving in particular that they are all Abelian. The results of this section also act as a motivation for the more general case discussed in Section 6.

Definition 5.1. We define the $n$-index $Q$-functions from the Kleinian $\sigma$-function as

$$
Q_{i_{1}, \ldots, i_{n}}=\left(-\frac{1}{2 \sigma^{2}}\right)\left(S \circ \mathcal{D}_{i_{1}} \circ \cdots \circ \mathcal{D}_{i_{n}}(\sigma \otimes \sigma)\right), \quad i_{1} \leq \cdots \leq i_{n} \in\{1, \ldots, g\} .
$$

We note that the indices on the $Q$-functions do not indicate differentiation, but rather the application of operators. The $n$ in the definition is just the number of operators applied, and is not related to the $n$ in an $(n, s)$-curve. Indeed, the results of this section and the next are independent of the underlying curve. This definition is equivalent to the following one more commonly found in the literature, using the Hirota derivative from the introduction

$$
Q_{i_{1}, i_{2}, \ldots, i_{n}}(\boldsymbol{u})=\left.\frac{(-1)}{2 \sigma(\boldsymbol{u})^{2}} D_{i_{1}} D_{i_{2}} \cdots D_{i_{n}} \sigma(\boldsymbol{u}+\boldsymbol{v}) \sigma(\boldsymbol{u}-\boldsymbol{v})\right|_{\boldsymbol{v}=\mathbf{0}} .
$$

We prefer Definition 5.1 as the notation generalises in a much simpler way.

We use the label $Q$ to follow the notation of Baker in [8], who defined the 4-index functions. The general definition above was developed in [21] and [20] when 6-index functions were needed to explicitly solve basis problems. Baker likely chose the notation $Q$ since the functions can be viewed as a generalisation of the 2 -index $\wp$-functions. Comparing with Definition 4.5 we see

$$
Q_{i j}=\frac{\sigma_{i} \sigma_{j}-\sigma \sigma_{i j}}{\sigma^{2}}=\wp_{i j} .
$$

We aim to prove that the $Q$-functions are Abelian. So we need to show that for $\ell \in \Lambda$

$$
Q_{i_{1}, i_{2}, \ldots, i_{n}}(\boldsymbol{u}+\boldsymbol{\ell})=Q_{i_{1}, i_{2}, \ldots, i_{n}}(\boldsymbol{u}) .
$$

We can check the periodicity of a 2-index $Q$-function by simply substituting in the quasiperiodicity property of $\sigma(\boldsymbol{u})$ to the expression in $\sigma$-derivatives above. While this approach can be used to prove the periodicity of $Q$-functions for other values of $n$, it does not extend to an inductive proof due to the symmetrization operator, $S$, in the definition. 
Corollary 2.4 shows us that the $n$-index $Q$-functions are zero when $n$ is odd. A similar calculation shows that for $n$ even the functions are divisible by two. Lemma 2.3 may be used to give the general formula for an $n$-index $Q$-function in $\sigma$-derivatives and we may show by induction that

$$
\sigma_{i_{1}, i_{2}, \ldots, i_{n}}(\boldsymbol{u}+\boldsymbol{\ell})=\chi e^{L} \sum_{m=0}^{n}\left(\sum_{1 \leq j_{1}<j_{2}<\cdots<j_{m} \leq n} \sigma_{I_{n} \backslash\left\{i_{j_{1}}, i_{j_{2}}, \ldots, i_{j_{m}}\right\}} L_{i_{j_{1}}} L_{i_{j_{2}}} \cdots L_{i_{j_{m}}}\right)
$$

using the fact that second derivatives of $L(\boldsymbol{u})$ are zero. However, substituting these properties into the formula for the $Q$-functions and observing the cancelations is not trivial.

Instead, we use the properties of the operators to prove the result using the following property of $h$, the extra factor introduced in the quasi-periodicity condition (4.4).

Lemma 5.2. We have $S \circ \mathcal{D}_{i_{1}} \circ \cdots \circ \mathcal{D}_{i_{n}}(h \otimes h)=0$ for all $n \geq 1$.

Proof. We note that if $n$ is odd then the result follows directly from Corollary 2.4. To show the result also holds for $n$ even consider Lemma 2.3 with $f=h$,

$$
S \circ \mathcal{D}_{i_{1}} \circ \cdots \circ \mathcal{D}_{i_{n}}(h \otimes h)=\sum_{m=0}^{n}(-1)^{m}\left(\sum_{1 \leq j_{1}<j_{2}<\cdots<j_{m} \leq n} h_{I_{n} \backslash\left\{i_{j_{1}}, i_{j_{2}}, \ldots, i_{j_{m}}\right\}} h_{i_{j_{1}}, i_{j_{2}}, \ldots, i_{j_{m}}}\right),
$$

Since all the second derivatives of $L$ are zero we have $h_{i}=\chi e^{L} L_{i}, h_{i j}=\chi e^{L} L_{i} L_{j}$, and by a simple induction,

$$
h_{i_{1}, \ldots, i_{n}}=\chi e^{L} L_{i_{1}} \cdots L_{i_{n}} .
$$

Note that every term in the inner sum in equation (5.3) above contains each index in $I_{n}$ exactly once. Hence each of those terms are equal to $\chi^{2} e^{2 L} L_{i_{1}} \cdots L_{i_{n}}$. The number of terms is the number of ways of choosing $m$ from $n$, the binomial coefficient. Hence

$$
S \circ \mathcal{D}_{i_{1}} \circ \cdots \circ \mathcal{D}_{i_{n}}(h \otimes h)=\chi^{2} e^{2 L} L_{i_{1}} \cdots L_{i_{n}} \sum_{m=0}^{n}(-1)^{m}\left(\begin{array}{c}
n \\
m
\end{array}\right) .
$$

The lemma then follows from

$$
\sum_{m=0}^{n}(-1)^{m}\left(\begin{array}{l}
n \\
m
\end{array}\right)=0
$$

We now use this result to give a simple proof of the periodicity of the $Q$-functions.

Theorem 5.3. The $n$-index $Q$-functions are all Abelian.

Proof. By Definition 4.6 of the lattice operator we see that the Abelian property is equivalent to showing

$$
\Lambda_{\ell} \circ Q_{i_{1}, \ldots, i_{n}}=Q_{i_{1}, \ldots, i_{n}} .
$$

Using the definition of the $Q$-functions in equation (5.1), Lemma 4.7 which concluded that $\Lambda_{\ell}$ commutes with the other operators, and the periodicity property of $\sigma$, we have

$$
\Lambda_{\ell} \circ Q_{i_{1}, \ldots, i_{n}}=\left(-\frac{1}{2 h^{2} \sigma^{2}}\right) S \circ \mathcal{D}_{i_{1}} \circ \cdots \circ \mathcal{D}_{i_{n}} \circ \Lambda_{\ell}(\sigma \otimes \sigma)
$$


where $h=\chi e^{L}$. We observe that under the multiplication operation for tensor products,

$$
\Lambda_{\ell}(\sigma \otimes \sigma)=(h \sigma \otimes h \sigma)=(h \otimes h)(\sigma \otimes \sigma) .
$$

So we may use the Leibniz rule from Corollary 2.6 to give

$$
\mathcal{D}_{i_{1}} \circ \cdots \circ \mathcal{D}_{i_{n}}(h \otimes h)(\sigma \otimes \sigma)=\sum_{k=0}^{n} \sum_{\pi \in \Pi} \mathcal{D}_{\pi_{1}}(h \otimes h) \mathcal{D}_{\pi_{2}}(\sigma \otimes \sigma) .
$$

When $k=0$ we see there is only one $\pi \in \Pi$ and that $\pi_{1}$ is the empty set. So the inner sum in equation (5.6) produces just one term,

$$
(h \otimes h) \mathcal{D}_{i_{1}} \circ \cdots \circ \mathcal{D}_{i_{n}}(\sigma \otimes \sigma) .
$$

When $k=1$ there are $n$ entries in $\Pi$ and in each case $\pi_{1}$ is of length one. Hence the inner sum in equation (5.6) produces $n$ terms

$$
\mathcal{D}_{i_{1}}(h \otimes h) \mathcal{D}_{I_{n} \backslash i_{1}}(\sigma \otimes \sigma)+\mathcal{D}_{i_{2}}(h \otimes h) \mathcal{D}_{I_{n} \backslash i_{2}}(\sigma \otimes \sigma)+\cdots+\mathcal{D}_{i_{n}}(h \otimes h) \mathcal{D}_{I_{n} \backslash i_{n}}(\sigma \otimes \sigma) .
$$

Every term here involves a Baker-Hirota operator applied to $(h \otimes h)$ and hence under symmetrization each term becomes zero by Lemma 5.2. Similarly, for all $k>0$ the inner sum in equation (5.6) produces terms which all involve $k$ operators applied to $(h \otimes h)$. Only the term from $k=0$ will not vanish under symmetrization. Hence we have

$$
S \circ \mathcal{D}_{i_{1}} \circ \cdots \circ \mathcal{D}_{i_{n}}((h \otimes h)(\sigma \otimes \sigma))=h^{2} S \circ \mathcal{D}_{i_{1}} \circ \cdots \circ \mathcal{D}_{i_{n}}(\sigma \otimes \sigma)
$$

and so equation (5.5) becomes

$$
\Lambda_{\ell} \circ Q_{i_{1}, \ldots, i_{n}}=\left(-\frac{1}{2 h^{2} \sigma^{2}}\right) h^{2} S \circ \mathcal{D}_{i_{1}} \circ \cdots \circ \mathcal{D}_{i_{n}}(\sigma \otimes \sigma)=Q_{i_{1}, \ldots, i_{n}} .
$$

We have already noted that the 2 -index $Q$-function is exactly the function $\wp_{i j}$. It is actually possible to express any $Q$-function as a polynomial in the $\wp$-functions. In [18] the authors showed that

$$
Q_{i j k \ell}=\wp_{i j k \ell}-2 \wp_{i j} \wp_{k \ell}-2 \wp_{i k} \wp_{j \ell}-2 \wp_{i \ell} \wp_{j k}
$$

and similarly in [21] that

$$
\begin{aligned}
& Q_{i j k l m n}=\wp_{i j k l m n}-2\left[\left(\wp_{i j} \wp_{k l m n}+\wp_{i k} \wp_{j l m n}+\wp_{i l} \wp_{j k m n}+\wp_{i m} \wp_{j k l n}+\wp_{i n} \wp_{j k l m}\right)\right. \\
& +\left(\wp_{j k} \wp_{i l m n}+\wp_{j l} \wp_{i k m n}+\wp_{j m} \wp_{i k l n}+\wp_{j n} \wp_{i k l m}\right)+\left(\wp_{k l} \wp_{i j m n}+\wp_{k m} \wp_{i j l n}+\wp_{k n} \wp_{i j l m}\right) \\
& \left.+\left(\wp_{l m} \wp_{i j k n}+\wp_{l n} \wp_{i j k m}\right)+\wp_{m n} \wp_{i j k l}\right]+4\left[\left(\wp_{i j} \wp_{k l} \wp_{m n}+\wp_{i j} \wp_{k m} \wp_{l n}+\wp_{i j} \wp_{k n} \wp_{l m}\right)\right. \\
& +\left(\wp_{i k} \wp_{j l} \wp_{m n}+\wp_{i k} \wp_{j m} \wp_{l n}+\wp_{i k} \wp_{j n} \wp_{l m}\right)+\left(\wp_{i l} \wp_{j k} \wp_{m n}+\wp_{i l} \wp_{j m} \wp_{k n}+\wp_{i l} \wp_{j n} \wp_{k m}\right) \\
& \left.+\left(\wp_{i m} \wp_{j k} \wp_{l n}+\wp_{i m} \wp_{j l} \wp_{k n}+\wp_{i m} \wp_{j n} \wp_{k l}\right)+\left(\wp_{i n} \wp_{j k} \wp_{l m}+\wp_{i n} \wp_{j l} \wp_{k m}+\wp_{i n} \wp_{j m} \wp_{k l}\right)\right] .
\end{aligned}
$$

It follows from Theorem 6.4 later that the general formula is

$$
Q_{i_{1}, \ldots, i_{n}}=\sum_{\rho \in P_{n}^{[2]}}(-2)^{\ell-1} \sum_{\pi \in \Pi(\rho)} \wp_{\pi},
$$

where $P_{n}^{[2]}$ is the set of partitions of $n$ using only even numbers and $\ell$ the length of a given partition $\rho$. As usual, $\Pi(\rho)$ is the set of all disjoint partitions of the set of indices into subsets of lengths given $\rho$. We denote the resulting subsets of $\pi$ by $\pi_{1}, \ldots, \pi_{\ell}$ and let $\wp_{\pi}$ be the product of $\wp$-functions with indices the subsets $\pi_{i}$. 


\section{$6 \mathcal{R}$-functions}

In this section we study a new class of functions, designed to generalise the $\wp$ and $Q$-functions in turn. Hence we have chosen to call them $\mathcal{R}$-functions.

Definition 6.1. We define the $n$-index $m$ th order $\mathcal{R}$-functions from the Kleinian $\sigma$-function, using the generalised Baker-Hirota derivatives of Definition 3.2, as

$$
\mathcal{R}_{i_{1}, \ldots, i_{n}}^{[m]}=\left(-\frac{1}{m \sigma^{m}}\right)\left(S \circ \mathcal{H}_{i_{1}}^{[m]} \circ \cdots \circ \mathcal{H}_{i_{n}}^{[m]} \bigotimes_{k=1}^{m} \sigma\right),
$$

where $i_{1} \leq \cdots \leq i_{n} \in\{1, \ldots, g\}$.

Like the $Q$-functions, the indices of $\mathcal{R}$-functions do not indicate differentiation, but rather the application of operators.

Corollary 3.5 shows us that the $n$-index $\mathcal{R}$-functions are zero unless $n$ is a multiple of $m$. We can also observe from Corollary 3.6 that for such $n$ the functions are not identically zero. From the definition it is clear that the $m$ th order $\mathcal{R}$-functions have poles of order at most $m$, occurring on the $\Theta$-divisor. The main result of this section will be a proof that these functions are also Abelian, and hence can be used to complete the basis for $\Gamma(m)$. Following the approach taken for the $Q$-functions in the last section, we will do this using the Leibniz rule for the operators and the effect of the operators applied to $h$, the extra factor introduced in the quasi-periodicity condition (4.4) for the $\sigma$-function.

Lemma 6.2. We have, for all $n \geq 1$,

$$
S \circ \mathcal{H}_{i_{1}}^{[m]} \circ \cdots \circ \mathcal{H}_{i_{n}}^{[m]} \bigotimes_{k=1}^{m} h=0 .
$$

Proof. We note that if $n$ is not a multiple of $m$ then the result follows directly from Corollary 3.5. In Lemma 5.2 we could show directly that the symmetrization of the original BakerHirota operators evaluated on $h$ gave zero due to well known properties of the binomial coefficient involved. We saw in Section 3.2 that for the general operators the coefficients involve not just multinomial coefficients but also Möbius functions and so we use a different approach.

First we recall Lemma 3.3, which allows us to conclude that

$$
\mathcal{H}_{i_{1}}^{[m]} \circ \cdots \circ \mathcal{H}_{i_{n}}^{[m]} \bigotimes_{k=1}^{m} h=\sum_{\ell_{n}}\left(c_{\ell_{n}} \bigotimes_{k=1}^{m} h_{\pi_{k, \ell}}\right) .
$$

Here $\ell_{n}$ is an index and the sum is finite with the number of terms depending on $n$. Each $\pi_{k, \ell}$ is a subset of the indices $I_{n}=\left\{i_{1}, \ldots, i_{n}\right\}$ such that $\pi_{1, \ell}, \ldots, \pi_{k, \ell}$ are a disjoint partition of $I_{n}$. We further recall equation (5.4) which stated that for the function $h$ we have $h_{i_{1}, \ldots, i_{n}}=$ $\chi e^{L} L_{i_{1}} \cdots L_{i_{n}}$. Hence each of these tensor products will symmetrize to give the same function,

$$
S\left(\bigotimes_{k=1}^{m} h_{\pi_{k, \ell}}\right)=\prod_{k=1}^{m} h_{\pi_{k, \ell}}=\chi^{m} e^{m L} L_{i_{1}} \cdots L_{i_{n}} .
$$

So we have

$$
S \circ \mathcal{H}_{i_{1}}^{[m]} \circ \cdots \circ \mathcal{H}_{i_{n}}^{[m]} \bigotimes_{k=1}^{m} h=\chi^{m} e^{m L} L_{i_{1}} \cdots L_{i_{n}}\left(\sum_{\ell_{n}} c_{\ell_{n}}\right) .
$$


We aim to show that the sum of constants $c_{\ell_{n}}$ in equation (6.3) is always zero, which would allow us to conclude the lemma. For the case $n=1$ we have

$$
\mathcal{H}_{i_{1}}^{[m]} \bigotimes_{k=1}^{m} h=\sum_{j=1}^{m} \zeta^{j-1} \partial_{i_{1}}^{[j]} \bigotimes_{k=1}^{m} h
$$

and so $\ell_{1}$ runs from 1 to $m$ with $c_{\ell_{1}}=\zeta^{\ell_{1}-1}$. Clearly the sum of these constants is zero since $\zeta$ is an $m$ th root of unity.

Now consider applying another Hirota operator to equation (6.2):

$$
\mathcal{H}_{i_{1}}^{[m]} \circ \cdots \circ \mathcal{H}_{i_{n+1}}^{[m]} \bigotimes_{k=1}^{m} h=\sum_{j=1}^{m} \zeta^{j-1} \partial_{i_{n+1}}^{[j]} \sum_{\ell_{n}} c_{\ell_{n}} \bigotimes_{k=1}^{m} h_{\pi_{k}}=\sum_{j=1}^{m} \zeta^{j-1} \sum_{\ell_{n}} c_{\ell_{n}} \partial_{i_{n+1}}^{[j]} \bigotimes_{k=1}^{m} h_{\pi_{k}} .
$$

Since

$$
h_{\pi_{k}, i_{n+1}}=L_{i_{n+1}} h_{\pi_{k}}
$$

we have that for all $j=1, \ldots, m$,

$$
S\left(\partial_{i_{n+1}}^{[j]} \bigotimes_{k=1}^{m} h_{\pi_{k}}\right)=\chi^{m} e^{m L} L_{i_{1}} \cdots L_{i_{n+1}}
$$

So we see that

$$
S \circ \mathcal{H}_{i_{1}}^{[m]} \circ \cdots \circ \mathcal{H}_{i_{n+1}}^{[m]} \bigotimes_{k=1}^{m} h=\chi^{m} e^{m L} L_{i_{1}} \cdots L_{i_{n+1}}\left(\sum_{j=1}^{m} \zeta^{j-1} \sum_{\ell_{n}} c_{\ell_{n}}\right) .
$$

Hence

$$
\sum_{\ell_{n+1}} c_{\ell_{n+1}}=\sum_{j=1}^{m} \zeta^{j-1} \sum_{\ell_{n}} c_{\ell_{n}}=0 .
$$

We can now prove the periodicity of the $\mathcal{R}$-functions.

Theorem 6.3. The $n$-index mth order $\mathcal{R}$-functions are all Abelian.

Proof. By Definition 4.6 of the lattice operator we see that the Abelian property is equivalent to showing

$$
\Lambda_{\ell} \circ \mathcal{R}_{i_{1}, \ldots, i_{n}}^{[m]}=\mathcal{R}_{i_{1}, \ldots, i_{n}}^{[m]} .
$$

Using the definition of the $\mathcal{R}$-functions in equation (6.1), Lemma 4.7 which concluded that $\Lambda_{\ell}$ commutes with the other operators, and the periodicity property of $\sigma$, we have

$$
\Lambda_{\ell} \circ \mathcal{R}_{i_{1}, \ldots, i_{n}}^{[m]}=\left(-\frac{1}{m h^{m} \sigma^{m}}\right) S \circ \mathcal{H}_{i_{1}}^{[m]} \circ \cdots \circ \mathcal{H}_{i_{n}}^{[m]} \circ \Lambda_{\ell} \bigotimes_{k=1}^{m} \sigma,
$$

where $h=\chi e^{L}$. We observe that under the multiplication operation for tensor products

$$
\Lambda_{\ell}\left(\bigotimes_{k=1}^{m} \sigma\right)=\bigotimes_{k=1}^{m} h \sigma=\left(\bigotimes_{k=1}^{m} h\right)\left(\bigotimes_{k=1}^{m} \sigma\right) \text {. }
$$


So we may use the Leibniz rule from Corollary 3.9 to give

$$
\mathcal{H}_{i_{1}}^{[m]} \circ \cdots \circ \mathcal{H}_{i_{n}}^{[m]} \bigotimes_{k=1}^{m} h \bigotimes_{k=1}^{m} \sigma=\sum_{\ell=0}^{n} \sum_{\pi \in \Pi} \mathcal{H}_{\pi_{1}}^{[m]}\left(\bigotimes_{k=1}^{m} h\right) \mathcal{H}_{\pi_{2}}^{[m]}\left(\bigotimes_{k=1}^{m} \sigma\right) .
$$

When $\ell=0$ we see there is only one $\pi \in \Pi$ and that $\pi_{1}$ is the empty set. So the inner sum in equation (6.5) produces just one term,

$$
\left(\bigotimes_{k=1}^{m} h\right) \mathcal{H}_{i_{1}}^{[m]} \circ \cdots \circ \mathcal{H}_{i_{n}}^{[m]}\left(\bigotimes_{k=1}^{m} \sigma\right)
$$

For all $\ell>0$ the inner sum in equation (5.6) produces terms which all involve $\ell$ operators applied to $\otimes_{k=1}^{m} h$. So from Lemma 6.2 , only the term from $\ell=0$ will not vanish under symmetrization. Hence we have

$$
S \circ \mathcal{H}_{i_{1}}^{[m]} \circ \cdots \circ \mathcal{H}_{i_{n}}^{[m]}\left(\bigotimes_{k=1}^{m} h\right)\left(\bigotimes_{k=1}^{m} \sigma\right)=h^{m} S \circ \mathcal{H}_{i_{1}}^{[m]} \circ \cdots \circ \mathcal{H}_{i_{n}}^{[m]}\left(\bigotimes_{k=1}^{m} \sigma\right)
$$

and so equation (6.4) becomes

$$
\Lambda_{\ell} \circ \mathcal{R}_{i_{1}, \ldots, i_{n}}^{[m]}=\left(-\frac{1}{m h^{m} \sigma^{m}}\right) h^{m} S \circ \mathcal{H}_{i_{1}} \circ \cdots \circ \mathcal{H}_{i_{n}} \bigotimes_{k=1}^{m} \sigma=R_{i_{1}, \ldots, i_{n}}^{[m]} .
$$

Since the $\mathcal{R}$-functions are Abelian we know they can be written algebraically using the Kleinian $\wp$-function. We make this relationship explicit in the next theorem.

Theorem 6.4. The $n$-index $m$ th order $\mathcal{R}$-functions may be expressed in Kleinian $\wp$-functions as

$$
\mathcal{R}_{i_{1}, \ldots, i_{n}}^{[m]}=\sum_{\rho \in P_{n}^{[m]}}(-m)^{\ell-1} \sum_{\pi \in \Pi(\rho)} \wp_{\pi},
$$

where $P_{n}^{[m]}$ is the set of partitions of $n$ using only integers divisible by $m$ and $\ell$ the length of a given partition $\rho$. As usual, $\Pi(\rho)$ is the set of all disjoint partitions of the set of indices into subsets of lengths given $\rho$. We denote the resulting subsets of $\pi$ by $\pi_{1}, \ldots, \pi_{\ell}$ and let $\wp_{\pi}$ be the product of $\wp$-functions with indices the subsets $\pi_{i}$.

For example, in the $m=3$ case we have

$$
\begin{aligned}
\mathcal{R}_{i_{1}, i_{2}, i_{3}, i_{4}, i_{5}, i_{6}}^{[3]}= & \wp_{i_{1}, i_{2}, i_{3}, i_{4}, i_{5}, i_{6}}-3\left(\wp_{i_{1}, i_{2}, i_{3}, \wp} \wp_{i_{4}, i_{5}, i_{6}}+\wp_{i_{1}, i_{2}, i_{4}} \wp_{i_{3}, i_{5}, i_{6}}+\wp_{i_{1}, i_{2}, i_{5}} \wp_{i_{3}, i_{4}, i_{6}}\right. \\
& +\wp_{i_{1}, i_{2}, i_{6}} \wp_{i_{3}, i_{4}, i_{5}}+\wp_{i_{1}, i_{3}, i_{4}} \wp_{i_{2}, i_{5}, i_{6}}+\wp_{i_{1}, i_{3}, i_{5}} \wp_{i_{2}, i_{4}, i_{6}}+\wp_{i_{1}, i_{3}, i_{6}} \wp_{i_{2}, i_{4}, i_{5}} \\
& \left.+\wp_{i_{1}, i_{4}, i_{5}} \wp_{i_{2}, i_{3}, i_{6}}+\wp_{i_{1}, i_{4}, i_{6}} \wp_{i_{2}, i_{3}, i_{5}}+\wp_{i_{1}, i_{5}, i_{6}} \wp_{i_{2}, i_{3}, i_{4}}\right) .
\end{aligned}
$$

Note that equation (6.6) clearly reduces to equation (5.7) in the $m=2$ case.

Proof. In this proof we define a symbol $\hat{\wp}$ such that $\sigma=e^{\hat{\wp}}$. Then $\hat{\wp}=\log (\sigma), \hat{\wp}_{i}=\sigma_{i} / \sigma$ and

$$
\hat{\wp}_{i_{1}, \ldots, i_{n}}=-\wp_{i_{1}, \ldots, i_{n}}
$$

for $n \geq 2$. It is important to note that $\hat{\wp}$ is not the Weierstrass $\wp$-function and neither $\hat{\wp}$ nor $\hat{\wp}_{i}$ are Abelian functions. We consider an $m$ th order $\mathcal{H}$-operator acting on a tensor product of $\sigma$.

$$
\mathcal{H}_{i_{1}}^{[m]} \bigotimes_{k=1}^{m} \sigma=\mathcal{H}_{i_{1}}^{[m]} \bigotimes_{k=1}^{m} e^{\hat{\wp}}=\sum_{j=1}^{m} \zeta^{j-1} \partial_{i}^{[j]} \bigotimes_{k=1}^{m} e^{\hat{\wp}} .
$$


This will be a sum of terms, each a tensor product in which every entry has a factor $e^{\hat{\wp}}$. So using the multiplication for tensor products, we have

$$
\mathcal{H}_{i_{1}}^{[m]} \bigotimes_{k=1}^{m} \sigma=\left(\sum_{j=1}^{m} \zeta^{j-1}\left(1 \otimes 1 \otimes \cdots \otimes \hat{\wp}_{i_{1}} \otimes 1 \otimes \cdots \otimes 1\right)\right)\left(\bigotimes_{k=1}^{m} e^{\hat{\wp}}\right)
$$

with the $\hat{\wp}_{i_{1}}$ occurring in the $j$ th position.

For any subset $I \in I_{n}=\left\{i_{1}, \ldots, i_{n}\right\}$ define

$$
\Sigma_{I}=\sum_{j=1}^{m} \zeta^{|I|(j-1)}\left(1 \otimes 1 \otimes \cdots \otimes \hat{\wp}_{I} \otimes 1 \otimes \cdots \otimes 1\right) .
$$

Then equation (6.7) becomes

$$
\mathcal{H}_{i_{1}}^{[m]}\left(\bigotimes_{k=1}^{m} \sigma\right)=\Sigma_{\left\{i_{1}\right\}}\left(\bigotimes_{k=1}^{m} \sigma\right) .
$$

Now consider applying a second operator. Using the product rule for $\mathcal{H}$-operators from Lemma 3.8 we have

$$
\mathcal{H}_{i_{1}}^{[m]} \circ \mathcal{H}_{i_{2}}^{[m]}\left(\bigotimes_{k=1}^{m} \sigma\right)=\mathcal{H}_{i_{2}}^{[m]}\left(\Sigma_{\left\{i_{1}\right\}}\right)\left(\bigotimes_{k=1}^{m} \sigma\right)+\Sigma_{\left\{i_{1}\right\}} \mathcal{H}_{i_{2}}^{[m]}\left(\bigotimes_{k=1}^{m} \sigma\right) .
$$

Applying the $\mathcal{H}$-operator on $\Sigma_{\left\{i_{1}\right\}}$ will produce a sum of terms, many of which will have zero as one of the entries in the tensor product. In this proof we will be applying operators and then symmetrizing, meaning that these terms will all vanish in the end and so we need not keep track of them. We let the symbol $\doteq$ indicate not a true equality, but that the difference consists only of terms with zero as one of the entries in the tensor product. When we apply the $\mathcal{H}$-operator on $\Sigma_{I}$ the only terms which will not vanish are those where the differentiation is applying to the entry with $\hat{\wp}_{I}$. We hence have

$$
\mathcal{H}_{i_{k}}^{[m]}\left(\Sigma_{I}\right) \doteq \Sigma_{\left\{I, i_{k}\right\}} .
$$

This result, along with (6.8), simplify equation (6.9) to

$$
\mathcal{H}_{i_{1}}^{[m]} \circ \mathcal{H}_{i_{2}}^{[m]}\left(\bigotimes_{k=1}^{m} \sigma\right) \doteq \Sigma_{\left\{i_{1}, i_{2}\right\}}\left(\bigotimes_{k=1}^{m} \sigma\right)+\Sigma_{\left\{i_{1}\right\}} \Sigma_{\left\{i_{2}\right\}}\left(\bigotimes_{k=1}^{m} \sigma\right) .
$$

An induction gives

$$
\mathcal{H}_{i_{1}}^{[m]} \circ \cdots \circ \mathcal{H}_{i_{n}}^{[m]}\left(\bigotimes_{k=1}^{m} \sigma\right) \doteq \sum_{\rho \in P_{n}} \sum_{\pi \in \Pi(\rho)} \Sigma_{\pi}\left(\bigotimes_{k=1}^{m} \sigma\right),
$$

where $P_{n}^{[m]}$ is the set of partitions of $n$ and the other notation as stated in the theorem.

Now consider the application of the symmetrization operator. We have

$$
\left.S\left(\bigotimes_{k=1}^{m} \sigma\right)=\sigma^{m} \quad \text { and } \quad S\left(\Sigma_{I}\right)=\hat{\wp}_{I}\left(\sum_{j=1}^{m} \zeta^{|I|(j-1)}\right)\right) .
$$

As in the proof of Lemma B.3, if $m$ divides $|I|$ then we obtain

$$
S\left(\Sigma_{I}\right)=m \hat{\wp}_{I}=-m \wp_{I} .
$$


and obtain zero otherwise. Hence (6.10) becomes

$$
S \circ \mathcal{H}_{i_{1}}^{[m]} \circ \cdots \circ \mathcal{H}_{i_{n}}^{[m]}\left(\bigotimes_{k=1}^{m} \sigma\right)=\sum_{\rho \in P_{n}^{[m]}}(-m)^{\ell} \sum_{\pi \in \Pi(\rho)} \wp_{\pi} \sigma^{m}
$$

and dividing by $-m \sigma^{m}$ gives the result of the theorem.

It is clear from their definitions that the $Q$-functions are the 2 nd order $\mathcal{R}$-functions:

$$
Q_{i_{1}, i_{2}, \ldots, i_{n}}(\boldsymbol{u})=\mathcal{R}_{i_{1}, i_{2}, \ldots, i_{n}}^{[2]}(\boldsymbol{u}) .
$$

Hence in turn the two-index $\wp$-functions are the 2 -index 2 nd order $\mathcal{R}$-functions:

$$
\wp_{i, j}(\boldsymbol{u})=\mathcal{R}_{i, j}^{[2]}(\boldsymbol{u})
$$

However, we see from the following corollary that in fact all the Kleinian $\wp$-functions are themselves $\mathcal{R}$-functions.

Corollary 6.5. The $m$-index $m$ th order $\mathcal{R}$-functions are exactly the $m$-index $\wp$-functions,

$$
\wp_{i_{1}, i_{2}, \ldots, i_{m}}(\boldsymbol{u})=\mathcal{R}_{i_{1}, i_{2}, \ldots, i_{m}}^{[m]}(\boldsymbol{u}) .
$$

Proof. This is the application of Theorem 6.4 in the case $n=m$.

Recall that the $n$-index $\wp$-functions had definite parity indicated by $n$; i.e. they are odd if $n$ is odd and even if $n$ is even. This property extends to the $\mathcal{R}$-functions, and hence the $Q$-functions also.

Lemma 6.6. The $n$-index $\mathcal{R}$-functions have parity equal to that of $n$, with respect to the change of variables $\boldsymbol{u} \rightarrow[-1] \boldsymbol{u}$.

The proof is simple but lengthy by considering the different combinations of parity of the components. The full details are in Appendix C.

\section{New bases of Abelian functions}

In this section we present some new bases of Abelian functions, completed using $\mathcal{R}$-functions. These are for the two canonical genus three cases. In this section we work with functions of only three variables and so we drop the commas between indices for brevity. Since the genus is fixed so are the dimensions of corresponding bases. However the actual bases themselves require different functions, made apparent by the different weights in the two cases (see Section 4.2). We note that like the $\wp$-functions, the weights of the $\mathcal{R}$-functions are the sum of the weights of the variables indicated by the indices. This can be seen by considering $\mathcal{R}$-functions as a sum of terms, each with $\sigma^{m}$ in the denominator and a monomial of $\sigma$-derivatives in the numerator, which includes all the indices. The weight of $\sigma$-derivatives is the weight of $\sigma$ minus the weight of the indices and in each term the weight of $\sigma$ cancels leaving the overall weight determined only by the indices.

For each $\Gamma(m)$ a basis is constructed systematically as follows. We start by including the basis for the preceding case so we then need only search for functions with poles of order $m$. We consider a set of possible basis functions, say $\mathcal{R}$-functions with a given number of indices, and proceed in ascending weight order. We use the $\sigma$-expansion for the corresponding $(n, s)$-curve to see if a given function is linearly independent of the existing basis entries and if so add it to the basis. It is important to include the functions from the preceding bases when testing 
linear independence, since although the $m$ th order $\mathcal{R}$-functions have poles of order at most $m$, they may in fact have lower order poles when associated with certain curves and this can only be checked using the expansion. Once the set has been exhausted, we consider another set if necessary.

We use the weight theory to consider only linear combinations of functions (and curve parameters) which give the correct weight. We then substitute in sufficient terms of the expansion and see if there are solutions for the coefficients. Such calculations can be lengthly and grow in $\mathrm{CPU}$ time and memory requirement with both the genus and the number of poles. Significant computational simplifications can be made by writing procedures that take further advantage of the weight structure present in the theory. For example, when expanding the product of series it is only necessary to multiply those terms which will give the correct final weight, as the other terms must all cancel. We can further reduce calculations by noting that there is a finite weight range that basis entries can take. Entries in $\Gamma(m)$ can have weight no lower than $-m$ wt $(\sigma)$ (see Lemma 3.4 in [16]).

The bases presented below are all for functions associated with general $(n, s)$-curves as in equation (4.1). However, we only need use the series expansions associated to the corresponding cyclic curves in equation (4.2) to check linear independence. If an element cannot be expressed using the basis with the restriction on the parameters, then neither will it be expressible with the wider set of parameters. Further, we only need to use sufficient expansion to give non-zero evaluations of the functions considered in order to check whether they are linearly independent.

We note that while these bases are not unique, (they can formulated using different functions), their weight structures are. Also, there are other invariants of the bases, such as the number of odd and even functions. We have several conjectures on these invariants, which are still under investigation.

\subsection{Hyperelliptic curves}

The hyperelliptic curve of genus 2 is a special case as its bases can be finitely generated by differentiation (see Table 1 ). If desired, they could be reformulated using $\mathcal{R}$-functions. All the $\wp$-functions are themselves $\mathcal{R}$-functions and the extra function, $\Delta \in \Gamma(3)$, may be replaced by

the six index $\mathcal{R}$-function, $\mathcal{R}_{122222}^{[3]}$. Of course, $\Delta$ may be expressed as a linear combination of the basis entries. In the cyclic case the relationship is,

$$
\Delta=-\frac{2}{5} \lambda_{4} \mathcal{R}_{11}^{[2]}+\frac{4}{5} \lambda_{4}^{2} \mathcal{R}_{12}^{[2]}-\frac{7}{5} \lambda_{3} \mathcal{R}_{12}^{[2]}-\frac{1}{20} \mathcal{R}_{122222}^{[3]}-\frac{9}{5} \lambda_{1}
$$

In the genus 3 hyperelliptic case we find that a basis for $\Gamma(2)$ is

$$
\left\{1, \mathcal{R}_{11}^{[2]}, \mathcal{R}_{12}^{[2]}, \mathcal{R}_{13}^{[2]}, \mathcal{R}_{22}^{[2]}, \mathcal{R}_{23}^{[2]}, \mathcal{R}_{33}^{[2]}, \mathcal{R}_{2222}^{[2]}\right\}
$$

We note that $\mathcal{R}_{2222}^{[2]}$ is a second order $\mathcal{R}$-function, and so equal to $Q_{2222}$ as defined in Section 5 . We note further that there are two other 4-index $Q$-functions of this weight; $Q_{1133}$ and $Q_{1223}$ and either of these may be used instead. Indeed, there are even more alternative $Q$-functions that could play the role if we allow more indices.

We now proceed to give a basis for $\Gamma(3)$. We arrange the elements in the set as vertical lists purely to make the structure (number of functions of each type) clearer to the reader

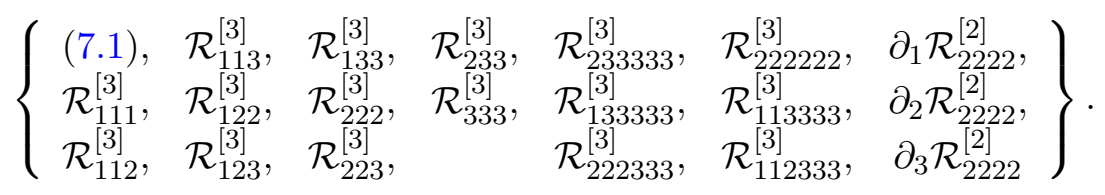


Here (7.1) is representing the eight entries in the basis for $\Gamma(2)$ presented in equation (7.1). We note that the three first derivatives of $\mathcal{R}_{2222}^{[2]}$ could be replaced by 9 -index third order $\mathcal{R}$-functions if desired. Finally, a basis for $\Gamma(4)$ is

$$
\left\{\begin{array}{cllll}
(7.2), & \mathcal{R}_{1223}^{[4]}, & \partial_{3} \mathcal{R}_{233333}^{[3]}, & \partial_{2} \mathcal{R}_{113333}^{[3]}, & \mathcal{R}_{12333333}^{[4]} \\
\mathcal{R}_{1111}^{[4]}, & \mathcal{R}_{1233}^{[4]}, & \partial_{2} \mathcal{R}_{233333}^{[3]}, & \partial_{1} \mathcal{R}_{1133}^{[3]}, & \mathcal{R}_{11333333}^{[4]}, \\
\mathcal{R}_{1112}^{[4]}, & \mathcal{R}_{1333}^{[4]}, & \partial_{3} \mathcal{R}_{133333}^{[3]}, & \partial_{2} \mathcal{R}_{112333}^{[3]}, & \mathcal{R}_{11233333}^{[4]}, \\
\mathcal{R}_{1113}^{[4]}, & \mathcal{R}_{2222}^{[4]}, & \partial_{1} \mathcal{R}_{233333}^{[3]}, & \partial_{3} \mathcal{R}_{222222}^{[3]}, & \mathcal{R}_{12223333}^{[4]}, \\
\mathcal{R}_{1122}^{[4]}, & \mathcal{R}_{2223}^{[4]}, & \partial_{2} \mathcal{R}_{133}^{[3]}, & \partial_{1} \mathcal{R}_{11233}^{[3]}, & \mathcal{R}_{11133333}^{[4]}, \\
\mathcal{R}_{1123}^{[4]}, & \mathcal{R}_{2233}^{[4]}, & \partial_{1} \mathcal{R}_{133}^{[3]}, & \partial_{2} \mathcal{R}_{222222}^{[3]}, & \mathcal{R}_{22222222}^{[4]}, \\
\mathcal{R}_{1133}^{[4]}, & \mathcal{R}_{2333}^{[4]}, & \partial_{2} \mathcal{R}_{222333}^{[3]}, & \partial_{1} \mathcal{R}_{222222}^{[3]}, & \mathcal{R}_{11123333}^{[4]} \\
\mathcal{R}_{1222}^{[4]}, & \mathcal{R}_{3333}^{[4]}, & \partial_{1} \mathcal{R}_{222333}^{[3]}, & &
\end{array}\right\} .
$$

In this case those first derivatives of third order $\mathcal{R}$-functions could not be replaced by any fourth order $\mathcal{R}$-functions. We may conclude this because previous calculations in [16] have shown that the basis must contain some odd functions with poles of order 4 . However, since the number of indices on the 4 th order $\mathcal{R}$-functions must be a multiple of 4 , all these functions must be even by Lemma 6.6. The odd functions with poles of order 4 are all found within the derivatives of the previous basis.

Although we have not proved that all bases in this case can be constructed this way, we have overcome one of the key difficulties in constructing such bases, which becomes apparent when examining the weights. In this case the variables $\boldsymbol{u}=\left(u_{1}, u_{2}, u_{3}\right)$ have weights $(5,3,1)$ while the $\sigma$-function has weight 6 . A basis for $\Gamma(m)$ must have an entry with the minimal weight of $-m$ wt $(\sigma)$ (see Lemma 3.4 in [16]). So in this case each $\Gamma(m)$ requires a function of weight $-6 m$ in its basis and such a function cannot be a derivative of a function in $\Gamma(m-1)$. This is since such a function could have weight no lower than

$$
-6(m-1)-5=-6 m+1
$$

achieved by differentiating a minimal weight function in $\Gamma(m-1)$ by $u_{1}$. We can now always choose the minimal weight function in the basis of $\Gamma(m)$ to be the $2 m$-index function $\mathcal{R}_{22 \ldots 2}^{[m]}$.

\subsection{Trigonal curves}

The trigonal curves are those $(n, s)$-curves with $n=3$. The simplest is the $(3,4)$-curve which also has genus three, but is non-hyperelliptic. We find that a basis for $\Gamma(2)$ is

$$
\left\{1, \mathcal{R}_{11}^{[2]}, \mathcal{R}_{12}^{[2]}, \mathcal{R}_{13}^{[2]}, \mathcal{R}_{22}^{[2]}, \mathcal{R}_{23}^{[2]}, \mathcal{R}_{33}^{[2]}, \mathcal{R}_{2222}^{[2]}\right\}
$$

We note that symbolically, this looks the same as equation (7.1). However, the two theories differ in the weights of the functions. In the hyperelliptic case $\boldsymbol{u}=\left(u_{1}, u_{2}, u_{3}\right)$ had weight $(5,3,1)$ while in the trigonal case the weights are $(5,2,1)$.

Once again, $\mathcal{R}_{2222}^{[2]}=Q_{2222}$ and there were other choices of $Q$-function. This time there was only one alternative 4-index $Q$-function of this weight, $Q_{1333}$, contrary to the alternatives in the hyperelliptic case, making the symbolic equivalence somewhat coincidental. However, there is also a structural equivalence in the use of six 2 -index functions and one 4 -index function.

For $\Gamma(3)$ in the trigonal case we find the basis

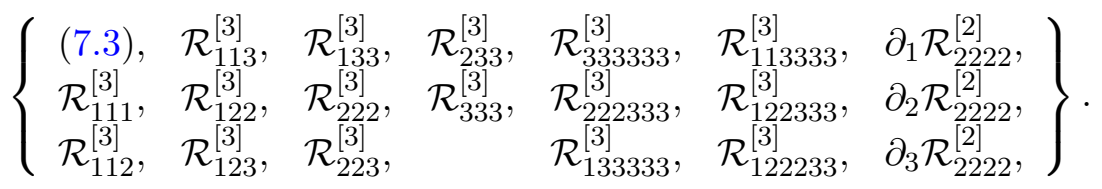


So the symbolic equivalence is now lost; for example the function $\mathcal{R}_{333333}^{[3]}$ is present in this basis but can not replace any function in the basis (7.2). However, the structural equivalence is still present; i.e. in both the hyperelliptic and trigonal cases the number of functions with a given number of indices was the same. Similarly, for $\Gamma(4)$ in the trigonal case we find the basis

$$
\left\{\begin{array}{cllll}
(7.4), & \mathcal{R}_{1223}^{[4]}, & \partial_{3} \mathcal{R}_{333333}^{[3]}, & \partial_{2} \mathcal{R}_{122333}^{[3]}, & \mathcal{R}_{2233333}^{[4]}, \\
\mathcal{R}_{1111}^{[4]}, & \mathcal{R}_{1233}^{[4]}, & \partial_{2} \mathcal{R}_{333333}^{[3]}, & \partial_{1} \mathcal{R}_{133}^{[3]}, & \mathcal{R}_{22233333}^{[4]}, \\
\mathcal{R}_{1112}^{[4]}, & \mathcal{R}_{1333}^{[4]}, & \partial_{3} \mathcal{R}_{222333}^{[3]}, & \partial_{2} \mathcal{R}_{122233}^{[3]}, & \mathcal{R}_{13333333}^{[4]}, \\
\mathcal{R}_{1113}^{[4]}, & \mathcal{R}_{2222}^{[4]}, & \partial_{1} \mathcal{R}_{333333}^{[3]}, & \partial_{2} \mathcal{R}_{113333}^{[3]}, & \mathcal{R}_{12233333}^{[4]}, \\
\mathcal{R}_{1122}^{[4]}, & \mathcal{R}_{2223}^{[4]}, & \partial_{2} \mathcal{R}_{222333}^{[3]}, & \partial_{1} \mathcal{R}_{122333}^{[3]}, & \mathcal{R}_{12223333}^{[4]}, \\
\mathcal{R}_{1123}^{[4]}, & \mathcal{R}_{2233}^{[4]}, & \partial_{2} \mathcal{R}_{133}^{[3]}, & \partial_{1} \mathcal{R}_{122}^{[3]}, & \mathcal{R}_{11333333}^{[4]}, \\
\mathcal{R}_{1133}^{[4]}, & \mathcal{R}_{2333}^{[4]}, & \partial_{3} \mathcal{R}_{122333}^{[3]}, & \partial_{1} \mathcal{R}_{113333}^{[3]}, & \mathcal{R}_{11223333}^{[4]} \\
\mathcal{R}_{1222}^{[4]}, & \mathcal{R}_{3333}^{[4]}, & \partial_{1} \mathcal{R}_{222333}^{[3]}, & &
\end{array}\right\}
$$

Again, the number of functions of each type is the same. We note that although bases for these spaces have been derived before in [18] and [16], this structural similarity only becomes apparent now, when using $\mathcal{R}$-functions.

We conjecture that in general a basis for $\Gamma(m)$ can always be constructed using the preceding basis, $m$ th order $\mathcal{R}$-functions and the first derivatives of $(m-1)$ th order $\mathcal{R}$-functions, which would solve the problem of identifying enough suitable basis functions.

\section{A Proof of Lemma 2.3}

We need to verify equation (2.1),

$$
\mathcal{D}_{i_{1}} \circ \cdots \circ \mathcal{D}_{i_{n}}(f \otimes g)=\sum_{m=0}^{n}(-1)^{m}\left(\sum_{1 \leq j_{1}<j_{2}<\cdots<j_{m} \leq n} f_{I_{n} \backslash\left\{i_{j_{1}}, i_{j_{2}}, \ldots, i_{j_{m}}\right\}} \otimes g_{i_{j_{1}}, i_{j_{2}}, \ldots, i_{j_{m}}}\right) .
$$

Proof. We give an inductive proof. First we observe the case $n=1$ where

$$
\begin{aligned}
\operatorname{RHS}(2.1) & =(-1)^{0}\left(f_{I_{1} \backslash \varnothing} \otimes g_{\varnothing}\right)+(-1)^{1}\left(\sum_{j_{1}=1}^{1} f_{I_{1} \backslash\left\{i_{j_{1}}\right\}} \otimes g_{i_{j_{1}}}\right) \\
& =f_{I_{1}} \otimes g_{\varnothing}-f_{\varnothing} \otimes g_{i_{1}}=f_{i_{1}} \otimes g-f \otimes g_{i_{1}}
\end{aligned}
$$

as required. We now assume the result holds for $n=k$ and consider the case $n=k+1$.

$$
\begin{aligned}
& \mathcal{D}_{i_{1}} \circ \cdots \circ \mathcal{D}_{i_{k+1}}(f \otimes g)=\mathcal{D}_{i_{k+1}} \circ \mathcal{D}_{i_{1}} \cdots \circ \mathcal{D}_{i_{k}}(f \otimes g) \\
& =\mathcal{D}_{i_{k+1}}\left(\sum_{m=0}^{k}(-1)^{m}\left(\sum_{1 \leq j_{1}<j_{2}<\cdots<j_{m} \leq k} f_{I_{k} \backslash\left\{i_{j_{1}}, i_{j_{2}}, \ldots, i_{j_{m}}\right\}} \otimes g_{i_{j_{1}}, i_{j_{2}}, \ldots, i_{j_{m}}}\right)\right) \\
& =\sum_{m=0}^{k}(-1)^{m}\left(\left(\sum_{1 \leq j_{1}<j_{2}<\cdots<j_{m} \leq k} f_{I_{k+1} \backslash\left\{i_{j_{1}}, i_{j_{2}}, \ldots, i_{j_{m}}\right\}} \otimes g_{i_{j_{1}}, i_{j_{2}}, \ldots, i_{j_{m}}}\right)\right. \\
& \left.-\left(\sum_{1 \leq j_{1}<j_{2}<\cdots<j_{m} \leq k} f_{I_{k} \backslash\left\{i_{j_{1}}, i_{j_{2}}, \ldots, i_{j_{m}}\right\}} \otimes g_{i_{j_{1}}, i_{j_{2}}, \ldots, i_{j_{m}}, i_{k+1}}\right)\right) .
\end{aligned}
$$


We need to show that equation (A.1) leads to

$$
\mathcal{D}_{i_{1}} \circ \cdots \circ \mathcal{D}_{i_{k+1}}(f \otimes g)=\sum_{m=0}^{k+1}(-1)^{m}\left(\sum_{1 \leq j_{1}<j_{2}<\cdots<j_{m} \leq k+1} f_{I_{k+1} \backslash\left\{i_{j_{1}}, i_{j_{2}}, \ldots, i_{j m}\right\}} \otimes g_{i_{j_{1}}, i_{j_{2}}, \ldots, i_{j_{m}}}\right) .
$$

Each value of $m$ in the outer sum in equation (A.1) gives two parts. The first part will contribute to one value of $m$ in equation (A.2) with the second contributing to the following value. These two parts are required to fill each value of $m$ in equation (A.2), with the exception of the first and last terms in the sum, for which one part is sufficient.

Consider the contribution of the first part of equation (A.1) when $m=0$. We obtain $f_{I_{k+1}} \otimes g$ which is what we require for $m=0$ in equation (A.2).

Similarly consider the contribution of the second part of equation (A.1) when $m=k$. We obtain $(-1)^{k+1} f \otimes g_{I_{k+1}}$ which is what we require for $m=k+1$ in equation (A.2).

Now we consider the general term. We combine the contribution of the first part of equation (A.1) when $m=m$ with the contribution of the second part when $m=m-1$ to obtain

$$
\begin{aligned}
& (-1)^{m}\left(\sum_{1 \leq j_{1}<j_{2}<\cdots<j_{m} \leq k} f_{I_{k+1} \backslash\left\{i_{j_{1}}, i_{j_{2}}, \ldots, i_{j_{m}}\right\}} \otimes g_{i_{j_{1}}, i_{j_{2}}, \ldots, i_{j_{m}}}\right. \\
& \left.\quad+\sum_{1 \leq j_{1}<j_{2}<\cdots<j_{m} \leq k} f_{I_{k} \backslash\left\{i_{j_{1}}, i_{j_{2}}, \ldots, i_{j_{m-1}}\right\}} \otimes g_{i_{j_{1}}, i_{j_{2}}, \ldots, i_{j_{m-1}}, i_{k+1}}\right) .
\end{aligned}
$$

Note that the minus sign in the second part was combined with the $(-1)^{m-1}$ outside to factor a $(-1)^{m}$ out altogether.

We can check that the terms here are referring to the same partition of the set of indices over the tensor product. Counting in the first part we see we have $k+1-m$ indices and $m$ indices. Similarly in the second part we have $k-(m-1)$ indices and $m-1+1$ indices, the same values.

Now note that the terms in the second part never contain $i_{k+1}$ on the left of the tensor product, but always contain it on the right. Hence these terms are those extra ones we would obtain if we ran the sum in the first part to $k+1$ instead of $k$. So these terms can be combined into the one sum to give,

$$
(-1)^{m}\left(\sum_{1 \leq j_{1}<j_{2}<\cdots<j_{m} \leq k+1} f_{I_{k+1} \backslash\left\{i_{j_{1}}, i_{j_{2}}, \ldots, i_{j_{m}}\right\}} \otimes g_{i_{j_{1}}, i_{j_{2}}, \ldots, i_{j_{m}}}\right),
$$

the term in equation (A.2) when $m=m$.

\section{B Symmetric functions}

We gather together some definitions and results for symmetric functions which we require.

Definition B.1. The monomial symmetric polynomials in $m$ variables, $x=\left[x_{1}, \ldots, x_{m}\right]$ are each defined by a sequence of natural numbers of length $m$, say $\rho=\left[\rho_{1}, \ldots, \rho_{m}\right]$, as

$$
M_{\rho}(x)=\sum_{\psi \in \Psi(\rho)} x^{\psi}
$$

Following the notation introduced for Lemma 3.3, $\Psi(\rho)$ is the set of all permutations of $\rho$ with entries denoted by $\rho=\left[\rho_{1}, \ldots, \rho_{m}\right]$. We then let $x^{\psi}$ denote a corresponding monomial,

$$
x^{\psi}=x_{1}^{\psi_{1}} x_{2}^{\psi_{2}} \cdots x_{m}^{\psi_{m}} .
$$


We define the augmented monomial symmetric polynomials as

$$
\hat{M}_{\rho}(x)=\sum_{\psi \in \Psi(x)} \psi^{\rho}
$$

This time the sum is over permutations of the variables rather than permutations of the powers.

For example,

$$
\begin{aligned}
M_{[3,1,1]}\left(x_{1}, x_{2}, x_{3}\right) & =x_{1}^{3} x_{2} x_{3}+x_{1} x_{2}^{3} x_{3}+x_{1} x_{2} x_{3}^{3}, \\
\hat{M}_{[3,1,1]}\left(x_{1}, x_{2}, x_{3}\right) & =x_{1}^{3} x_{2} x_{3}+x_{1}^{3} x_{3} x_{2}+x_{2}^{3} x_{1} x_{3}+x_{2}^{3} x_{3} x_{1}+x_{3}^{3} x_{1} x_{2}+x_{3}^{3} x_{2} x_{1} \\
& =2 M_{[3,1,1]}\left(x_{1}, x_{2}, x_{3}\right) .
\end{aligned}
$$

Let $\eta_{i}$ be the multiplicity of $i \in \rho$, i.e. the number of entries in $\rho$ equal to $i$. Then we have

$$
\hat{M}_{\rho}(x)=\left(\prod_{i} \eta_{i} !\right) M_{\rho}(x) .
$$

The monomial symmetric functions naturally occur when considering the application of $\mathcal{H}$ operators in Section 3.2. They occur evaluated on the roots of unity. Symmetric polynomials in the roots of unity have been considered previously in [30], with nice results for some of the other classes. For example, the elementary symmetric polynomials reduce to

$$
e_{1}=0, e_{2}=0, \ldots, e_{m-1}=0, e_{m}=(-1)^{m+1} \text {. }
$$

However, in our case, the power sum basis is the most useful.

Definition B.2. The Newton power sums in $m$ variables $x_{1}, \ldots, x_{m}$ are defined for $k \geq 0$ as

$$
p_{k}\left(x_{1}, \ldots, x_{m}\right)=x_{1}^{k}+x_{2}^{k}+\cdots+x_{m}^{k} .
$$

Any symmetric polynomial in $m$ variables can be expressed as a polynomial with rational coefficients in $p_{1}, \ldots, p_{m}$. We observe how they evaluate on our choice of variables.

Lemma B.3. The power sums evaluated on $X=\left[X_{1}, \ldots, X_{m}\right]$ where $X_{k}=\zeta^{k-1}$ for $\zeta$ a primitive mth root of unity satisfy

$$
p_{k}= \begin{cases}m & \text { if } m \mid k, \\ 0 & \text { otherwise }\end{cases}
$$

Proof. We have

$$
p_{k}(X)==1+\zeta^{k}+\zeta^{2 k}+\cdots+\zeta^{(m-1) k}=\frac{1-\zeta^{k m}}{1-\zeta^{k}} .
$$

Since $\zeta$ is an $m$ th root of unity, the numerator is always zero. The denominator is only non-zero for $m \mid k$. Hence if $k$ is not a multiple of $m$ then $p_{k}=0$. If $k=m$ then we have

$$
p_{k}=X_{1}^{m}+X_{2}^{m}+\cdots+X_{m}^{m}=1^{m}+\zeta^{m}+\zeta^{2 m}+\cdots+\zeta^{(m-1) m}=m .
$$

Similarly if $m \mid k$ then we have a sum of $m$ terms, each $\zeta$ raised to a power divisible by $m$. Hence the polynomial evaluates to $m$. 
We now introduce a procedure for expressing symmetric functions in power sums. Although every symmetric function of $m$ variables may be expressed as a polynomial in the first $m$ power sums $p_{k}$, this explicit procedure will instead give an expression in the first $n$ power sums, where $n$ is the number partitioned by the sequence $\rho=\left[\rho_{1}, \ldots, \rho_{m}\right]$.

We again let $\Pi$ be a set of partitions of indices. This time it will be all disjoint partitions, $\pi$, of the set of indices $I_{m}=\left\{i_{1}, \ldots, i_{m}\right\}$ into subsets denoted by $\pi_{1}, \ldots, \pi_{\ell}$. Note that we do not restrict to a given number of subsets or specify their size. We see that $\ell$ will range from 1 , (when $\pi$ has just $\pi_{1}=I_{m}$ itself), to $m$ (when $\pi$ consists of the $m$ subsets with one index each). We define the associated Möbius function $\mu(\pi)$ by

$$
\mu(\pi)=(-1)^{m-\ell} \prod_{i=1}^{\ell}\left(\left|\pi_{i}\right|-1\right) !
$$

and refer readers to [35] for more information on such functions.

Next we define length $\ell$ vectors $\boldsymbol{\nu}=\left[\nu_{1}, \ldots, \nu_{\ell}\right] \in \mathbb{N}^{\ell}$ by

$$
\boldsymbol{\nu}(\boldsymbol{\rho}, \boldsymbol{\pi})=\left[\sum_{i_{j} \in \pi_{1}} \rho_{j}, \ldots, \sum_{i_{j} \in \pi_{\ell}} \rho_{j}\right] .
$$

Finally we define the function $p_{\boldsymbol{\nu}}$ as the product of power sum functions whose indices are determined by the values in the vector $\boldsymbol{\nu}$,

$$
p_{\nu}=p_{\nu_{1}} \cdots p_{\nu_{2}} \text {. }
$$

We can now state the following formula of Doubilet from [15],

$$
\hat{M}_{\rho}=\sum_{\pi \in \Pi} \mu(\pi) p_{\nu(\rho, \pi)} .
$$

So the augmented monomial symmetric functions have an integer polynomial expression in the power sums $p_{1}, \ldots, p_{n}$. The expression for the monomial symmetric functions will be the same but with a fractional multiplicative constant depending on the multiplicities of $\rho$ as described in equation (B.1).

We give an example in the case $m=3$. We consider the disjoint partitions of $I_{3}=\left\{i_{1}, i_{2}, i_{3}\right\}$. The set $\Pi$ has five elements:

$$
\begin{array}{llll}
\pi^{[1]}: & \pi_{1}^{[1]}=\left\{i_{1}, i_{2}, i_{3}\right\}, & & \\
\pi^{[2]}: & \pi_{1}^{[2]}=\left\{i_{1}, i_{2}\right\}, & \pi_{2}^{[2]}=\left\{i_{3}\right\}, & \\
\pi^{[3]}: & \pi_{1}^{[3]}=\left\{i_{1}, i_{3}\right\}, & \pi_{2}^{[3]}=\left\{i_{2}\right\}, & \\
\pi^{[4]}: & \pi_{1}^{[4]}=\left\{i_{2}, i_{3}\right\}, & \pi_{2}^{[4]}=\left\{i_{1}\right\}, & \\
\pi^{[5]}: & \pi_{1}^{[5]}=\left\{i_{1}\right\} & \pi_{2}^{[5]}=\left\{i_{2}\right\}, & \pi_{3}^{[5]}=\left\{i_{3}\right\} .
\end{array}
$$

The Möbius function then gives

$$
\begin{aligned}
& \mu\left(\pi^{[1]}\right)=(-1)^{3-1} 2 !=+2, \\
& \mu\left(\pi^{[2]}\right)=\mu\left(\pi^{[3]}\right)=\mu\left(\pi^{[4]}\right)=(-1)^{3-2} 1 ! 0 !=-1, \\
& \mu\left(\pi^{[5]}\right)=(-1)^{3-3}(0 !)^{3}=+1 .
\end{aligned}
$$

Hence any augmented monomial symmetric function in 3 variables may be expressed using the power sums in 3 variables as

$$
\hat{M}_{\rho}=2 p_{\rho_{1}+\rho_{2}+\rho_{3}}-p_{\rho_{1}+\rho_{2}} p_{\rho_{3}}-p_{\rho_{1}+\rho_{3}} p_{\rho_{2}}-p_{\rho_{2}+\rho_{3}} p_{\rho_{1}}-p_{\rho_{1}} p_{\rho_{2}} p_{\rho_{3}} .
$$


For example,

$$
\hat{M}_{[3,1,1]}=2 p_{5}-2 p_{4} p_{1}-p_{2} p_{3}+p_{3} p_{1}^{2} .
$$

Note that when we have entries in $\rho$ which are zero then we need to recall that $p_{0}=m$. So in the $m=3$ case we have, for example,

$$
\hat{M}_{[3,0,0]}=2 p_{3}-3 p_{3} p_{0}+p_{3} p_{0}^{2}=2 p_{3} .
$$

\section{Proof of Lemma 6.6}

We prove that the $\mathcal{R}$-functions have parity equal to that of $n$, the number of indices.

Proof. Think of the $n$-index $m$ th order $\mathcal{R}$-functions as

$$
\mathcal{R}_{i_{1}, \ldots, i_{n}}^{[m]}=\frac{\text { polynomial in } \sigma \text {-derivatives }}{\sigma^{m}} .
$$

Each term in the polynomial in the numerator is a monomial in $\sigma$ and its derivatives containing $n$-indices overall (see Lemmas 3.3 and 3.4). Recall that the $\sigma$-function is either odd or even. In the case of $(n, s)$-curves this can be determined from $(n, s)$ (see Lemma 4.4). We refer to derivatives of $\sigma$ with respect to an odd number of variables as $\sigma_{\mathrm{o}}$-derivatives and similarly $\sigma_{\mathrm{e}}$-derivatives if differentiated with respect to an even number of variables.

Suppose first that $\sigma$ is an even function and so the denominator of (C.1) is even. In this case the $\sigma_{\mathrm{o}}$-derivatives are odd and the $\sigma_{\mathrm{e}}$-derivatives even. If $n$ is odd then each term in the numerator of (C.1) must contain an odd number of $\sigma_{\mathrm{o}}$-derivatives. Similarly if $n$ is even then each term would have an even number. Hence the numerator, and therefore $\mathcal{R}_{i_{1}, \ldots, i_{n}}^{[m]}$, has parity the same as $n$.

Now suppose that $\sigma$ is an odd function. This time the denominator of (C.1) has parity equal to that of $m$, and it is the $\sigma_{\mathrm{e}}$-derivatives which are odd while the $\sigma_{\mathrm{o}}$-derivatives are even. In this case we need to be more careful. Note that each term in the numerator of (C.1) can be thought of as a product of $\sigma$-derivatives multiplied by the $\sigma$-function itself raised to a power.

If $n$ is odd then $m$ must be also, making the denominator of (C.1) odd. Each term in the numerator must contain an even number of $\sigma_{\mathrm{e}}$-derivatives, paired with an odd number of $\sigma_{\mathrm{o}^{-}}$ derivatives. Hence, since $m$ is odd, any $\sigma$-functions in the term must be raised to an even power. So overall each term in the numerator is even and hence $\mathcal{R}_{i_{1}, \ldots, i_{n}}^{[m]}$ is odd overall.

If $n$ is even then we must consider the two choices for $m$ separately. If $m$ is even then so is the denominator of (C.1). Each term in the numerator must have an even number of $\sigma$ derivatives overall. If there are an odd number of $\sigma_{\mathrm{e}}$-derivatives then there are an even number of $\sigma_{\mathrm{o}}$-derivatives and hence, since $m$ is even, a $\sigma$-function raised to an odd power. Then each term in the numerator is even overall. If there were an even number of $\sigma_{\mathrm{e}}$-derivatives then there are an odd number of $\sigma_{\mathrm{o}}$-derivatives and any remaining $\sigma$-function is raised to an even power. Either way, each term in the numerator is even overall and hence so is $\mathcal{R}_{i_{1}, \ldots, i_{n}}^{[m]}$. Finally, if $m$ were odd then the same analysis will this time show that each term in the numerator is odd. However, the denominator will also be odd in this case and so $\mathcal{R}_{i_{1}, \ldots, i_{n}}^{[m]}$ is still even overall.

\section{Acknowledgments}

We acknowledge Mr. Lachlan Walker who contributed some preliminary work to proving that $Q$-functions are Abelian. In particular he proved equation (5.2), and derived a formula for the 8 -index $Q$-functions in terms of $\wp$-functions which helped motivate equation (5.7). We would also like to thank the two anonymous referees for their useful comments. 


\section{References}

[1] Athorne C., A novel approach to the theory of Padé approximants, J. Nonlinear Math. Phys. 12 (2005), suppl. 2, 15-27.

[2] Athorne C., Algebraic Hirota maps, in Bilinear integrable systems: from classical to quantum, continuous to discrete, NATO Sci. Ser. II Math. Phys. Chem., Vol. 201, Springer, Dordrecht, 2006, 17-33.

[3] Athorne C., Algebraic invariants and generalized Hirota derivatives, Phys. Lett. A 256 (1999), 20-24.

[4] Athorne C., Identities for hyperelliptic $\wp$-functions of genus one, two and three in covariant form, J. Phys. A: Math. Theor. 41 (2008), 415202, 20 pages, arXiv:0808.3103.

[5] Athorne C., Eilbeck J.C., Enolskii V.Z., Identities for the classical genus two $\wp$ function, J. Geom. Phys. 48 (2003), 354-368.

[6] Baker H.F., Abelian functions: Abel's theorem and the allied theory of theta functions, Cambridge University Press, Cambridge, 1995.

[7] Baker H.F., Multiply periodic functions, Cambridge University Press, Cambridge, 2007.

[8] Baker H.F., On a system of differential equations leading to periodic functions, Acta Math. 27 (1903), $135-156$.

[9] Baldwin S., Eilbeck J.C., Gibbons J., Ônishi Y., Abelian functions for cyclic trigonal curves of genus 4, J. Geom. Phys. 58 (2008), 450-467, math.AG/0612654.

[10] Baldwin S., Gibbons J., Genus 4 trigonal reduction of the Benney equations, J. Phys. A: Math. Gen. 39 (2006), 3607-3639.

[11] Buchstaber V.M., Enolskii V.Z., Leykin D.V., Kleinian functions, hyperelliptic Jacobians and applications, Rev. Math. Math. Phys. 10 (1997), 1-125.

[12] Buchstaber V.M., Enolskii V.Z., Leykin D.V., Rational analogues of abelian functions, Funct. Anal. Appl. 33 (1999), 83-94.

[13] Cho K., Nakayashiki A., Differential structure of abelian functions, Internat. J. Math. 19 (2008), 145-171, math.AG/0604267.

[14] Dodd R.K., Eilbeck J.C., Gibbon J.D., Morris H.C., Solitons and nonlinear wave equations, Academic Press Inc., London, 1982.

[15] Doubilet P., On the foundations of combinatorial theory. VII. Symmetric functions through the theory of distribution and occupancy, Studies in Appl. Math. 51 (1972), 377-396.

[16] Eilbeck J.C., England M., Ônishi Y., Abelian functions associated with genus three algebraic curves, $L M S J$. Comput. Math. 14 (2011), 291-326, arXiv:1008.0289.

[17] Eilbeck J.C., Enolskii V.Z., Leykin D.V., On the Kleinian construction of abelian functions of canonical algebraic curves, in SIDE III - Symmetries and Integrability of Difference Equations (Sabaudia, 1998), CRM Proc. Lecture Notes, Vol. 25, Amer. Math. Soc., Providence, RI, 2000, 121-138.

[18] Eilbeck J.C., Enolski V.Z., Matsutani S., Ônishi Y., Previato E., Abelian functions for trigonal curves of genus three, Int. Math. Res. Not. (2007), Art. ID rnm 140, 38 pages, math.AG/0610019.

[19] England M., Deriving bases for Abelian functions, Comput. Meth. Funct. Theor. 11 (2011), 617-654, arXiv:1103.0468.

[20] England M., Higher genus Abelian functions associated with cyclic trigonal curves, SIGMA 6 (2010), 025, 22 pages, arXiv:1003.4144.

[21] England M., Eilbeck J.C., Abelian functions associated with a cyclic tetragonal curve of genus six, J. Phys. A: Math. Theor. 42 (2009), 095210, 27 pages, arXiv:0806.2377.

[22] England M., Gibbons J., A genus six cyclic tetragonal reduction of the Benney equations, J. Phys. A: Math. Theor. 42 (2009), 375202, 27 pages, arXiv:0903.5203.

[23] Enolski V.Z., Hackmann E., Kagramanova V., Kunz J., Lämmerzahl C., Inversion of hyperelliptic integrals of arbitrary genus with application to particle motion in general relativity, J. Geom. Phys. 61 (2011), 899-921, arXiv:1011.6459.

[24] Grammaticos B., Ramani A., Hietarinta J., Multilinear operators: the natural extension of Hirota's bilinear formalism, Phys. Lett. A 190 (1994), 65-70.

[25] Hietarinta J., Gauge symmetry and the generalization of Hirota's bilinear method, J. Nonlinear Math. Phys. 3 (1996), 260-265. 
[26] Hietarinta J., Grammaticos B., Ramani A., Integrable trilinear PDE's, in Nonlinear Evolution Equations \& Dynamical Systems: NEEDS '94 (Los Alamos, NM), World Sci. Publ., River Edge, NJ, 1995, 54-63, solv-int/9411003.

[27] Hirota R., The direct method in soliton theory, Cambridge Tracts in Mathematics, Vol. 155, Cambridge University Press, Cambridge, 2004.

[28] Korotkin D., Shramchenko V., On higher genus Weierstrass sigma-function, Phys. D, to appear, arXiv:1201.3961.

[29] Lang S., Introduction to algebraic and abelian functions, Graduate Texts in Mathematics, Vol. 89, 2nd ed., Springer-Verlag, New York, 1982.

[30] Lascoux A., Schützenberger M.P., Formulaire raisonne de fonctions symetriques, Universite Paris 7, 1985.

[31] Matsumura H., Commutative algebra, W.A. Benjamin, Inc., New York, 1970.

[32] McKean H., Moll V., Elliptic curves: function theory, geometry, arithmetic, Cambridge University Press, Cambridge, 1997.

[33] Nakayashiki A., On algebraic expressions of sigma functions for ( $n, s)$ curves, Asian J. Math. 14 (2010), 175-211, arXiv:0803.2083.

[34] Olver P.J., Sanders J.A., Transvectants, modular forms, and the Heisenberg algebra, Adv. in Appl. Math. 25 (2000), 252-283.

[35] Rota G.C., On the foundations of combinatorial theory. I. Theory of Möbius functions, Z. Wahrscheinlichkeitstheorie und Verw. Gebiete 2 (1964), 340-368.

[36] Washington L.C., Elliptic curves: number theory and cryptography, 2nd ed., Discrete Mathematics and its Applications (Boca Raton), Chapman \& Hall/CRC, Boca Raton, FL, 2008. 